\title{
ACTVITY CLASSIFICATION IN USERS OF ANKLE FOOT ORTHOSES
}

\author{
A Thesis \\ Presented to \\ the faculty of the School of Engineering and Applied Science \\ University of Virginia
}

\author{
In partial fulfillment \\ of the requirements for the degree \\ Master of Science in Mechanical Engineering
}

by

Cameron M Archer

May 2013 


\section{APPROVAL SHEET}

The thesis

is submitted in partial fulfillment of the requirements

for the degree of

Master of Science in Mechanical Engineering

Cameron M Archer

AUTHOR

The thesis has been read and approved by the examining committee:

Bradford C Bennett

Thesis Advisor

Silvia S Blemker

John C Lach

Accepted for the School of Engineering and Applied Science:

James H Aylor

Dean, School of Engineering and Applied Science

May 2013 


\begin{abstract}
New technology in motion sensing has allowed for an advance in gait analysis and cerebral palsy diagnostics. Body sensors networks have emerged as a promising tool for gait analysis and activity recognition, and orthotic treatment is prevalent among those with cerebral palsy. In this work, a framework for activity classification using inertial sensors mounted on ankle foot orthoses (AFOs) is presented. A hybrid decision treenearest neighbor algorithm classifies activities and postures using subject-specific training. To evaluate sensitivities, eight volunteer subjects wore modified bilateral AFOs with shank and foot mounted triaxial accelerometers and gyroscopes. The AFOs were fitted with hardware to induce different gait perturbations: free rotation of the ankle, plantarflexion or "equinus" gait, and locked ankle joint. For each condition, the subject performed eight gait activities at varied slopes and standing, sitting, and lying postures.

Using data from the test protocols, the classification framework was performed to assess training data and number of nearest neighbor effects on classification sensitivity. These tests showed high sensitivity levels even with training data which did not outsize the test data, and that the highest sensitivities were obtained using only one nearest neighbor for comparison. Using optimal training data size and one nearest neighbor methods, forced activity classification was performed using the classification framework to assess sensitivity results for each activity. The results from these tests indicated high levels of sensitivity in recognizing predefined gait events for all perturbed conditions, and that semi-natural movement could be classified to some degree using annotated, predefined
\end{abstract}


movement for training. Subsequently, feature selection using cluster analysis was explored, indicating that feature reduction based on significance thresholds improved results for semi-natural activity classification. Finally, a declassification metric was examined, and results showed increased specificity results using declassification. Our results indicate that AFOs are a suitable sensor platform for future research in activity classification and gait monitoring in AFO users with perturbed gait using limited training data. 


\section{Table of Contents}

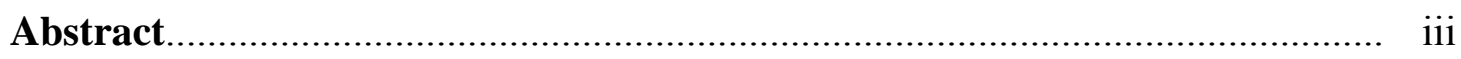

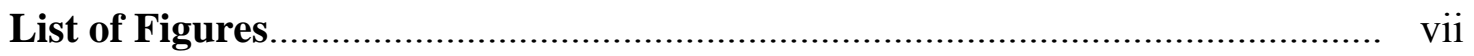

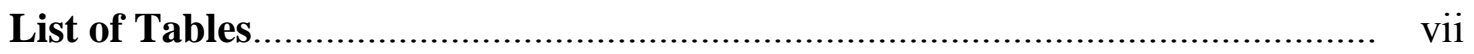

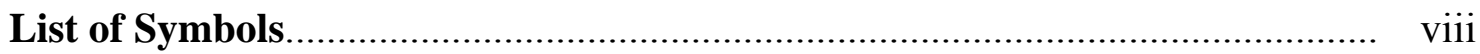

1.0 Introduction: Diagnosis, Assessment, and Treatment of Cerebral Palsy... 1

1.1 Cerebral Palsy and Treatment Options................................................. 1

1.2 Motion Capture for Assessing CP Gait.................................................. 2

1.3 Ankle Foot Orthoses in CP Treatment................................................... 3

1.4 Overview............................................................................... 4

2.0 Background: Gait Biomechanics in Cerebral Palsy and Activity Classification using Inertial Measurement Units........................................ $\quad 5$

2.1 Gait Biomechanics.......................................................................... 5

2.1.1 The Gait Cycle.................................................................... 5

2.1.2 Gait Abnormalities in $\mathrm{CP}$ and the Effects of AFO Use......... 7

2.2 Accelerometry and Inertial Measurement Units................................... 8

2.2.1 Accelerometers and Gyroscopes.............................................. 8

2.2.2 TEMPO 3 Inertial Measurement Units................................... 9

2.3 Data Mining and Activity Classification.............................................. 11

2.3.1 Hierarchical Decision Trees................................................. 12

2.3.2 Gaussian Mixture Models......................................................... 14

2.3.3 Hidden Markov Models........................................................ 15

2.3.4 k-Nearest Neighbor Models.................................................... 16

2.3.5 Artificial Neural Networks.................................................. 17

2.3.6 Selection of Data Mining Technique for This Work............. 18

3.0 Activity Classification of Healthy Subjects Wearing Test AFOs................ 21

3.1 Custom AFOs to Induce Gait Perturbation.......................................... 21

3.2 Activity Test Protocol...................................................................... 23

3.3 Signal Processing and Data Reduction............................................... 27

3.3.1 Data Windowing............................................................... 27

3.3.2 Kinematic Feature Reduction............................................... 28

3.3.3 Notes on the Feature Set..................................................... 37

3.4 Data Training and Distance Calculation............................................ 38

3.4.1 Partitioning Training Data..................................................... 38

3.4.2 Nearest Neighbor Distance Calculation................................. 39

3.5 Results for Forced Classification......................................................... 41

3.5.1 Sensitivity as a Function of Training Data Size.................... 42

3.5.2 Sensitivity as a Function of k................................................ 44 
3.5.3 Forced Classification at $\mathrm{k}=1,50 \%$ Training Data............. 46

3.6 Feature Selection Using Cluster Analysis..................................... 51

3.6.1 Clustering in the Training Data......................................... 52

3.6.2 Results with Feature Selections.......................................... 53

3.7 Introducing a Declassification Distance Metric................................. 56

3.7.1 Calculation of the Distance Metric................................... 57

3.7.2 Results with Varied Scaling Factor.................................. 58

3.7.3 Intermediate Classification............................................... 59

4.0 Conclusions and Recommendations for Future Work.......................... 62

4.1 Summary of Research................................................................... 62

4.2 Recommendations for Future Work........................................... 64

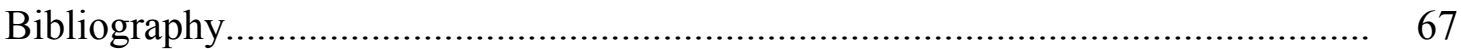




\section{List of Figures}

Figure 1: The gait cycle........................................................................... 6

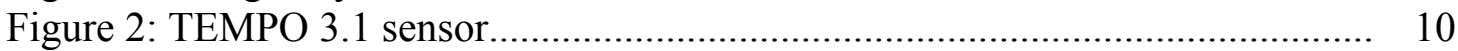

Figure 3: Custom decision tree structure........................................................ 13

Figure 4: Hidden Markov model structure......................................................... 15

Figure 5: kNN classification model........................................................... 17

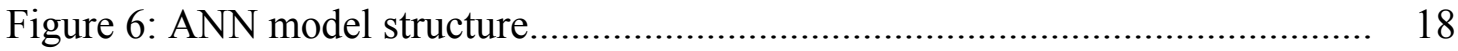

Figure 7: Classification framework structure.................................................. 19

Figure 8: Results from test AFO walking perturbation study............................... 23

Figure 9: Subject wearing test AFOs with hardware and optical markers.............. 25

Figure 10: Sample TEMPO data for walking-running transition............................ 26

Figure 11: Standard deviation of acceleration during walking and standing........... $\quad 30$

Figure 12: Gravitational axial components of the TEMPO sensors........................ 31

Figure 13: Maximum rotational velocity axial components for activities.............. 32

Figure 14: Maximum sagittal-plane shank angles for activities........................... 33

Figure 15: Ankle angle validation with Vicon ${ }^{\circledR}$ optical data............................... 34

Figure 16: Sum of maximum and minimum foot velocity for stairs..................... 35

Figure 17: Velocity correction methods............................................................. 36

Figure 18: Sensitivity results with increasing training data................................. 43

Figure 19: Sensitivity results with $k=1,3$, and 5........................................ 45

Figure 20: Sensitivity results for all activities at $\mathrm{k}=1,50 \%$ training................... 49

Figure 21: Usage percentage of features using cluster feature selection.................. 54

Figure 22: Sensitivity results for increasing selectivity of features...................... 55

Figure 23: Results for increasing declassification threshold.............................. 59

Figure 24: Occurrence of intermediate classifications.................................... 60

\section{List of Tables}

Table 1: List of kinematic features used in study............................................ 28

Table 2: Sensitivity results for different distance metrics................................... 41

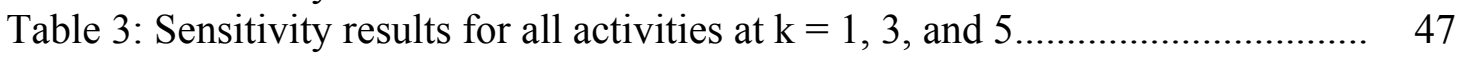

Table 4: Conglomerate confusion matrix for all activities.................................. 48 


\section{List of Symbols}

\begin{tabular}{|c|c|}
\hline$a_{z}$ & Vertical acceleration of the foot \\
\hline $\mathrm{c}$ & Feature magnitude of the cluster center \\
\hline $\mathrm{d}$ & Unscaled coordinate distance vector between training and test windows \\
\hline $\mathrm{D}$ & The standardized Euclidean distance between a training and test window \\
\hline$d^{\prime}$ & Scaled coordinate distance vector between training and test windows \\
\hline $\mathrm{D}_{\text {out }}$ & The distance of an outlier \\
\hline FN & Number of false negatives \\
\hline FP & Number of false positives \\
\hline h & Declassification metric scaling factor \\
\hline 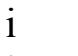 & Feature number \\
\hline & Cluster number \\
\hline $\mathrm{k}$ & Number of nearest neighbors used for classification \\
\hline 1 & Number of clusters \\
\hline $\mathrm{m}$ & Number of features \\
\hline $\mathrm{n}$ & Sample number \\
\hline $\mathrm{N}_{\mathrm{b}}$ & Number of windows for a particular cluster classification \\
\hline $\mathrm{N}_{\mathrm{d}}$ & Number of distances calculated to determine declassification threshold \\
\hline $\mathrm{N}_{\mathrm{q}}$ & Number of data windows accurately clustered \\
\hline $\mathrm{N}_{\mathrm{s}}$ & Number of classification subsets \\
\hline $\mathrm{p}$ & Cluster precision \\
\hline $\mathrm{Q}_{1}$ & The first quartile of the training data distances \\
\hline $\mathrm{Q}_{3}$ & The third quartile of the training data distances \\
\hline $\mathbf{S}$ & Vector of the variances of each feature \\
\hline $\mathrm{t}$ & Feature selection precision threshold \\
\hline $\mathrm{TN}$ & Number of true negatives \\
\hline $\mathrm{TP}$ & Number of true positives \\
\hline $\mathrm{u}$ & Cluster weighting value \\
\hline $\mathrm{v}_{\mathrm{z}}$ & Vertical velocity of the foot \\
\hline $\mathrm{w}_{\mathrm{b}}$ & Number of windows in a classification subset \\
\hline $\mathrm{w}_{\mathrm{c}}$ & Number of data windows in a cluster \\
\hline $\mathrm{W}_{\mathrm{r}}$ & Number of training data windows \\
\hline $\mathrm{W}_{\mathrm{s}}$ & Number of test data windows \\
\hline $\mathrm{x}$ & Scalar feature value of the training data window \\
\hline $\mathbf{X}$ & Training data feature matrix \\
\hline $\mathrm{X}_{\mathrm{b}}$ & Training data feature matrix for a classification subset \\
\hline $\mathrm{y}$ & Scalar feature value of the test data window \\
\hline $\mathbf{Y}$ & Test data feature matrix \\
\hline$\Delta$ & Sampling period \\
\hline$\sigma$ & Standard deviation of the feature vector \\
\hline$\varphi$ & Sagittal-plane shank angle \\
\hline 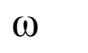 & Sagittal-plane rotation velocity of the shank \\
\hline
\end{tabular}




\subsection{Introduction: Diagnosis, Assessment, and Treatment of Cerebral}

\section{Palsy}

\subsection{Cerebral Palsy and Treatment Options}

Individuals with walking disabilities due to neuromuscular disorders such as cerebral palsy have recently benefitted from the development of new medical technologies which improve diagnostic capability. In particular, motion capture systems have provided clinicians with a diagnostic tool to quantitatively assess gait abnormalities in these individuals, and prescribe treatment in the form of surgical intervention, orthotic use, and/or physical therapy.

Cerebral palsy (CP) is a non-progressive disorder which affects movement and posture due to abnormal development or trauma of the motor control centers of the immature brain ${ }^{1}$. The disorder is characterized by a loss of gross motor function due to tightness of the muscles, muscle spasticity, and involuntary movement. This loss of motor control leads to gait abnormality. The presentation of gait abnormalities among those with $\mathrm{CP}$ is remarkably heterogeneous, however, and abnormalities characterized by the disorder are not limited to losses in motor function ${ }^{1}$. The United Cerebral Palsy Research and Education Foundation estimates between 1.5-2.0 million children and adults in the United States have CP, and around 11,000 are newly diagnosed each year ${ }^{2}$.

Many with CP deal with spasticity, an increase in the muscular stretch reflex which creates muscle tightness. Spasticity causes abnormalities in muscle tone, loss of coordination and balance, and impairment of selective motor control. Conservative 
treatments include physical therapy, occupational therapy, oral medication, and orthoses. These may be accompanied by invasive treatments such botulinum neurotoxin injection or surgeries such as orthopaedic surgery, intrathecal baclofen administration, or selective dorsal rhizotomy ${ }^{3}$. Different techniques have varied in their levels of success, though orthoses are generally regarded as successful and non-invasive treatment for those with spastic diplegia $^{4-7}$.

\subsection{Motion Capture for Assessing CP Gait}

Children with CP have benefitted from recent developments in medical technology as diagnostic approaches and interventions can be specifically tailored to individuals with unique disabilities. Treatments for spastic CP have been aimed at improving functionality while preventing the development of secondary problems such as contracture and bony deformity. Due to the heterogeneity of the disorder, however, treatment modalities must be considered through careful diagnostic methods ${ }^{8}$.

Currently, most clinical analysis of CP gait is accomplished by trained observers or physical therapists in a laboratory with optical motion-capture equipment. Motion capture systems provide physicians, therapists, and researchers a detailed description of a patient's gait kinematics including joint angles and range of motion, center of mass displacement, stride length, and cadence. The inclusion of force plates and EMG can provide kinetic measures of gait through the analysis of ground reaction forces. Though they provide clinicians with detailed information of patients' condition, infrared camera systems such as those made by Vicon ${ }^{\circledR}$ can cost hundreds of thousands of dollars and are 
generally not portable. They do not offer a long term assessment of the patient, but instead provide only a small sample of gait events per patient visit. Additionally, clinical analysis within a laboratory environment can restrict a patient's ability to reproduce a typical gait pattern over varied terrain. Children can be especially non-compliant in unfamiliar settings.

The difficulties that arise during motion capture analysis of CP gait led researchers at the Motion Analysis and Motor Performance Laboratory to assess the feasibility of employing mobile activity monitoring systems for studying CP gait. A mobile activity monitoring system using non-interfering, wireless, body-worn sensors could provide clinicians with a reliable long-term method for monitoring physical activity and gait patterns in patients outside of the clinic and give a realistic account of the patient's typical activity. Like optical motion capture systems, this system would still allow doctors to assess the results of intervention and therapy.

\subsection{Ankle-Foot Orthoses in CP Treatment}

Ankle-foot orthoses (AFOs) are frequently prescribed to those with spastic CP to improve motor function and stability and prevent the development of contractures. AFOs discourage abnormal gait patterns such as ankle plantarflexion or "equinus" gait and improve foot clearance during swing phase. Because they range in functional effect and can be custom fitted to patients, they are ideal for treating a variety of abnormalities caused by CP. Their use amongst ambulatory CP patients is widespread; over $50 \%$ of children with $\mathrm{CP}$ are prescribed $\mathrm{AFOs}^{9}$. Because they are a very common treatment for 
$\mathrm{CP}$, AFOs can provide a suitable platform for integrating treatment and diagnostics, providing feedback on functional improvement to the clinician.

This research evaluates the use of AFOs as a sensor mounting platform to be used for activity monitoring. The project aims to develop an activity classification routine capable of identifying periods of activity and posture in AFO users through the use of discreet sensors mounted on the AFO. This research could provide a significant improvement in out-of-laboratory analysis and diagnostics of gait in those with $\mathrm{CP}$, reducing the cost of analysis and improving patient quality of life.

\subsection{Overview}

Following this introduction, Chapter 2 provides background information on gait biomechanics, gait pathology in cerebral palsy, and activity classification using inertial measurement units. Chapter 3 describes the framework for activity classification using nearest neighbor methods, including feature reduction from raw lower limb kinematic data acquired through TEMPO sensors, training data selection effects, forced classification, feature selection effects, declassification metrics, and sub-classification clustering. Chapter 4 provides a summary of the research with conclusions about the impact of this work and recommendations for future research. 


\subsection{Background: Gait Biomechanics in Cerebral Palsy and Activity Classification using Inertial Measurement Units}

\subsection{Gait Biomechanics}

The activity classification routine discussed in this thesis work is designed to examine kinematic features from the movement of the user. The routine will use quantitative characteristics of gait and postures to classify the user's activity. This section presents information on the biomechanics of human gait, the gait pathologies that commonly present in $\mathrm{CP}$ and their effect on gait biomechanics, and how AFOs contribute to reduce the effect of these pathologies.

\subsubsection{The Gait Cycle}

The "gait cycle" is a term which describes the repetitive mechanical processes of the lower body which propel the body center of mass (COM) forward during walking or running. Because walking is the most efficient form of locomotion on level terrain, most humans have very similar gait patterns. The gait cycle encompasses a full stride, or a single step by both the left and right limbs, and consists of phases of double support, stance, and swing. Figure 1 shows a full human gait cycle. Double support phases are

defined by periods when both feet contact the ground. The stance phase for a limb is defined by the period when the foot touches the ground. The stance phase for each limb encompasses both the double support phase and the swing phase of the opposite limb. Swing phase for a limb is defined by the period when the foot is not in contact with the ground, and it coincides entirely with the stance phase of the opposite limb. Most 
clinicians choose the beginning of the gait cycle to be defined by foot contact of the forward limb. In this discussion, the initial forward limb will be the right leg. At this point the rearward (left) limb will still be in contact with the ground, so the gait cycle begins in an initial double support phase. During the initial double support phase, the anchoring of the right leg results in a deceleration of the COM, with most of the weight of the body shifting to the right $\operatorname{leg}^{10}$. As the weight shifts forward, the left leg supports less and less weight, and the left ankle alternates from dorsiflexion to plantarflexion. This plantarflexion of the rear foot provides the majority of the propulsive power in the gait cycle.

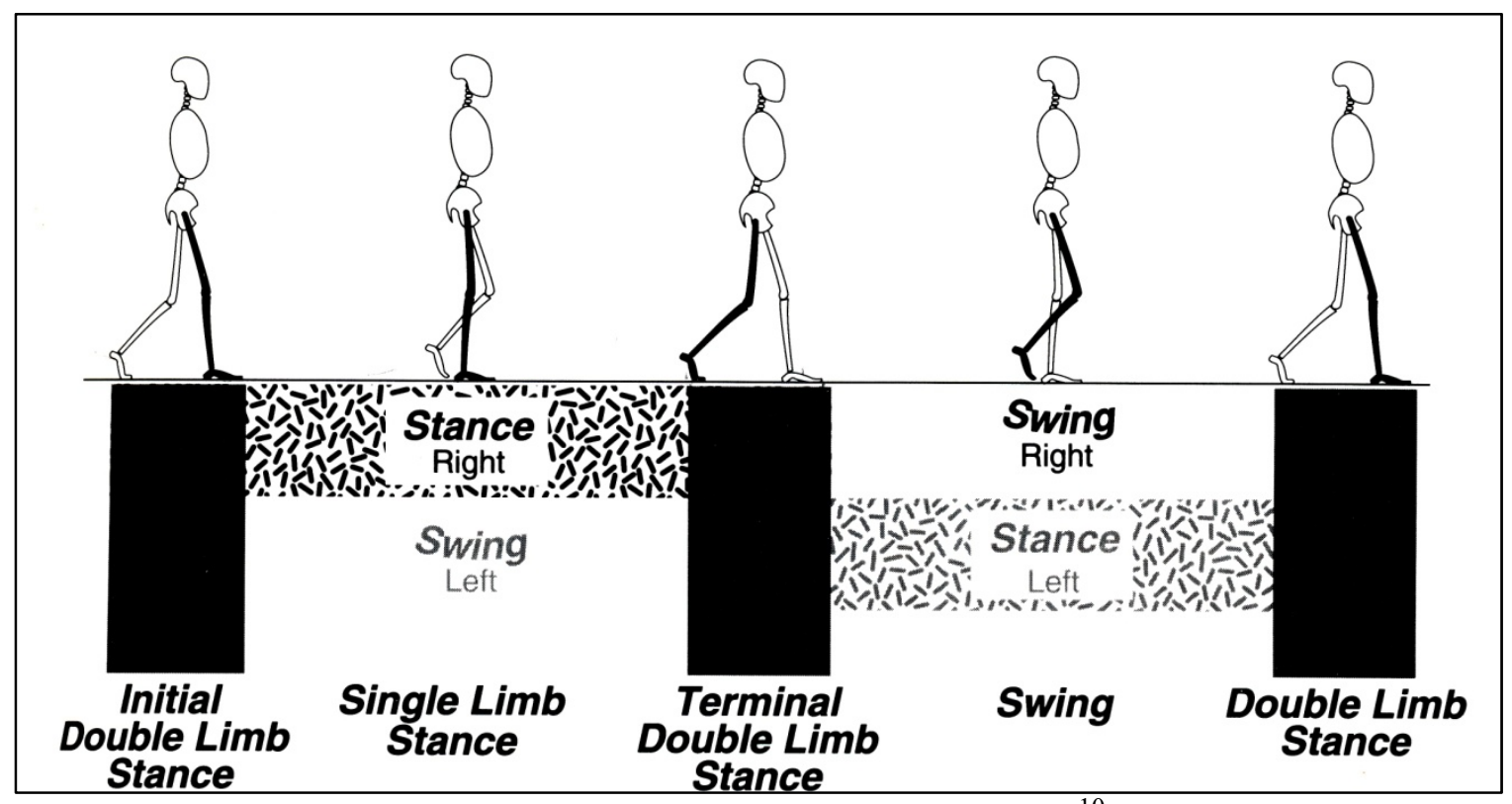

Figure 1. The complete human gait cycle ${ }^{10}$

The initial double support phase ends when the left foot loses contact with the ground. The swing phase of the left leg follows the initial double support phase. As the left foot lifts from the ground, the left knee flexes and the COM is raised as the supporting leg becomes more vertical. The lift off of the left leg initiates the single 
support phase for the right leg and the swing phase for the left leg. Once the left leg has swung through and contacts the ground the second, or terminal, double support phase begins. This phase is identical to the initial double support phase, but the limb mechanics are switched to the opposite limbs. The COM is again decelerated, this time by the anchoring of the left leg. Weight shifts forward as the right ankle switches from dorsiflexion to plantarflexion, again providing propulsive power. This phase ends when the right foot loses contact with the ground and begins its swing phase.

The swing phase of the right leg is identical to the swing phase of the left leg. The right knee flexes as the COM is raised through pelvic tilt and extension of the left knee to provide clearance for the right foot to swing forward. When the right foot leaves the ground, the single support phase for the left leg is initiated along with the swing phase for the right foot. When the right foot makes contact with the ground following swing phase, the gait cycle is completed.

\subsubsection{Gait Abnormalities in CP and the Effects of AFO Use}

Although the diagnosis of CP blankets a range of gait abnormalities, there are a few that are most common, especially among candidates for AFO prescription. A study by Wren et al. compares the prevalence of specific abnormalities in subjects with $\mathrm{CP}^{11}$. $61 \%$ of all CP patients in the study exhibited equinus gait, a condition which involves excessive plantarflexion of the ankle due to contraction of the posterior calf muscles. CP patients with equinus gait walk on their toes, with minimal or no heel contact with the ground. This presents several problems, including loss of power during push-off, instability during stance, and an overall loss of walking efficiency ${ }^{12}$. Another abnormality 
in CP is crouch gait. In the Wren et al. study, 69\% of subjects exhibited crouch gait, a condition defined by excessive knee flexion during the gait cycle. This excessive flexion can cause weakness in the ankle due to excessive dorsiflexion and may lead to secondary deformities $^{12}$. In both equinus and crouch gait conditions, patients are typically prescribed AFOs to provide support to the ankle and foot to preserve the lever forces required to propel the COM forward during walking ${ }^{12}$.

\subsection{Accelerometry and Inertial Measurement Units}

Activity recognition systems using body-worn sensors have been shown to allow for the detection and classification of a wide range of activities, in both supervised and unsupervised settings ${ }^{13-19}$. Most of these studies tested healthy subjects with normal gait cycles, but a few have used subjects with atypical gait. Classification schemes have been developed for the elderly ${ }^{13,16,20}$, trans-tibial amputees ${ }^{21}$, spine injury patients ${ }^{22,23}$, those with neurological disorders such as Parkinson's ${ }^{24,25}$, multiple sclerosis ${ }^{26}$, and $\mathrm{CP}^{27,28}$, or those with stimulation-assisted walking ${ }^{25,29}$. All of these studies used some combination of accelerometers and gyroscopes for activity classification or movement monitoring. This section describes the use of IMUs, including TEMPO 3 sensors, to acquire kinematic data.

\subsubsection{Accelerometers and Gyroscopes}

Inertial measurement units (IMUs) have typically been composed of accelerometers and/or gyroscopes. When coupled, these sensors provide measures of 
angular velocity and linear acceleration with as many as 6 degrees of freedom. IMUs can provide an improvement over traditional laboratory motion capture equipment, both for their mobility and low cost. They provide portable motion tracking at a minute fraction of the cost of motion capture equipment. They can be mounted discreetly within existing products or devices, and in some case provide real time feedback.

Despite these improvements, IMUs do introduce some challenges. Accelerometers and gyroscopes are sensitive to noise and drift errors. These errors can present significant issues when extracting gait features from the raw data, and they must be accounted for through filtering or data fusion techniques. For instance, we may desire to determine joint angle from a pair of IMUs. This would require integration of the gyroscope angular velocity signal. Even if the drift error in the gyroscope is small, it will be compounded through integration. So while IMUs appear to be the optimal means of mobile motion tracking, there are significant challenges to overcome.

\subsubsection{TEMPO 3 Inertial Measurement Units}

TEMPO (Technology-Enabled Medical Precision Observation) 3.1 and 3.2 are custom body sensor platforms designed and built by researchers at the University of Virginia's Center for Wireless Health. TEMPO body sensor networks (BSNs) incorporate sensors with tri-axis acceleromery and tri-axis gyroscopy for 6 degrees of freedom in the form factor of a wristwatch to enable kinematic data collection. TEMPO BSNs are attractive for use with AFOs because they are entirely wireless, capable of Bluetooth realtime data receiving (TEMPO 3.1), as well as long-term Flash memory storage (TEMPO 3.2f). Their small form factor allows them to be discreetly mounted within modified 
AFOs with no discomfort to the wearer. TEMPO 3.1 sensors incorporate a mixed-signal processor (TI MSP430F1611) to facilitate digital signal processing and a Bluetooth 2.0 transceiver (Roving Networks RN-41) for wireless communication to a PC or smart phone. Linear accelerations are captured with a Freescale MMA7261 three-axis, monolithic, micro-machined accelerometer. Figure 2 shows a TEMPO 3.1 sensor strapped to the subject ankle, and demonstrates the component axes of the accelerometer and gyroscope signals.

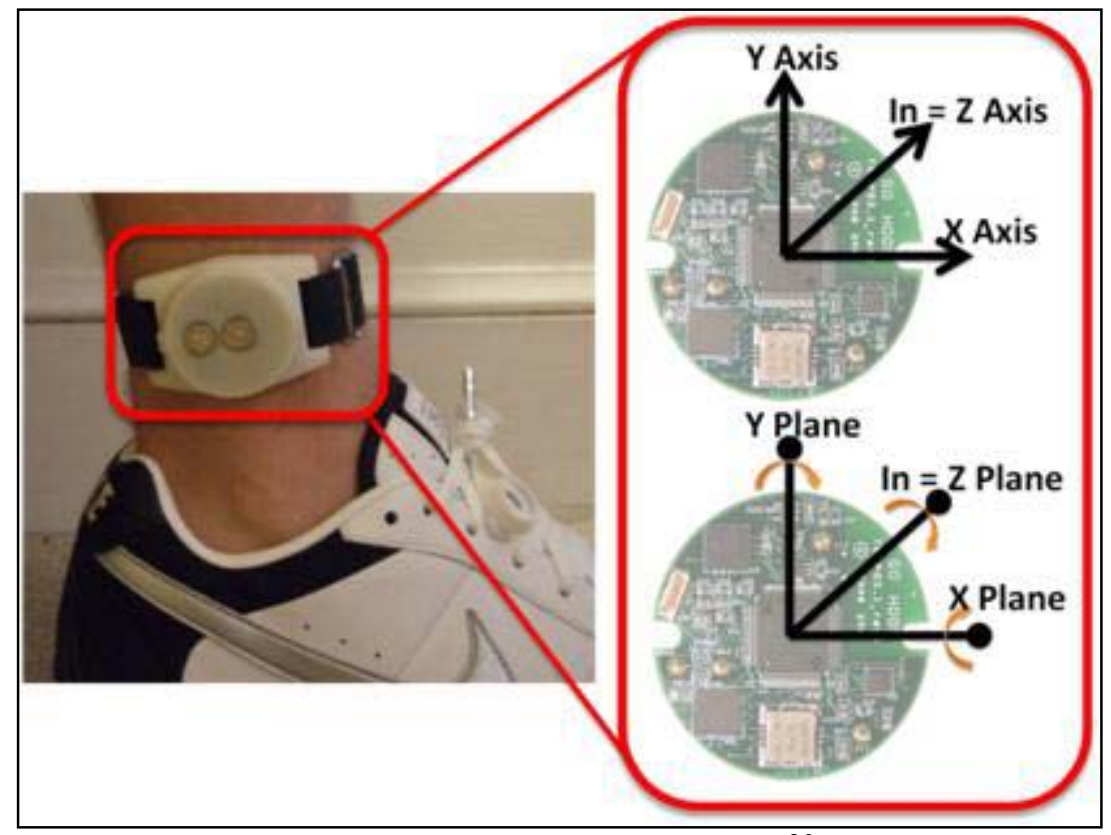

Figure 2. TEMPO 3.1 sensor $^{30}$

The accelerometer features selectable sensitivity between $\pm 2.5 \mathrm{~g}$ and $\pm 10 \mathrm{~g}$ to accommodate a wide range of human movement applications. Two micro-machined gyroscopes, the InvenSense IDG-300 and Analog Devices ADXRS610, capture triaxial angular velocity to at least \pm 300 degrees per second. Sensor outputs are conditioned by single-pole low-pass filters with $60 \mathrm{~Hz}$ cutoff frequencies. The six signals are captured by 12-bit analog to digital converter (ADC) channels. Conditioned signals are sampled by 
the ADC at $128 \mathrm{~Hz}$ - a bandwidth far exceeding the characteristic response of human movement. The MSP430 processor operates at $4 \mathrm{MHz}$ by a digitally-controlled oscillator synchronized to a low-power $32 \mathrm{kHz}$ crystal. The node is supplied by a rechargeable 3.7 $\mathrm{V}, 250 \mathrm{mAh}$ lithium-ion coin cell battery. For a complete introduction to TEMPO 3.1 BSNs, see the seminal work by Barth et $\mathrm{al}^{31}$. TEMPO $3.2 \mathrm{f}$ uses the same sensors and signal processes as TEMPO 3.1 but allows long-term data storage supplied via Flash memory.

TEMPO BSNs have been previously used in assessing human motion and gait pathology. TEMPO sensors have been used for clinical assessment of tremor in individuals with Parkinson's disease ${ }^{32}$, differentiating normal and "shuffling" gait ${ }^{33}$, fall detection $^{34}$, and spatio-temporal gait feature extraction ${ }^{30,35}$. These studies have demonstrated success in using TEMPO sensors to quantify and classify normal and pathological movement and gait, and provide additional support to the concept of AFO sensor mounting as a means of quantifying and classifying gait in AFO users such as children with CP.

\subsection{Data Mining and Activity Classification}

Most activity recognition schemes using IMUs involve comparing periods of data in which the activity is unknown to a set of training data. Algorithms are trained by selecting representative periods of activity data, usually collected by a supervisor, with annotated periods of known activities or postures such as walking, running, sitting or standing, etc. For these known activity periods, a set of kinematic features are reduced 
from the sensor data. Accelerometers and gyroscopes can provide a number of features such as orientation components, velocities, acceleration magnitudes and directions, and FFT components. Feature sets of future test data collections are then compared to the training data using data mining techniques, and periods of activity and posture are identified. The studies cited above have used several different classification algorithms. Promising statistical techniques include hierarchical decision trees ${ }^{14,16}$, Gaussian mixture models $^{13}$, hidden Markov models ${ }^{36,37}$, support vector machines ${ }^{38,39}$, nearest neighbor $\operatorname{models}^{14,40}$, and artificial neural networks ${ }^{41}$, as well as hybrid models incorporating multiple techniques ${ }^{15}$. This section describes the advantages and disadvantages of several common data mining techniques used in activity classification and outlines the technique used for this work.

\subsubsection{Hierarchical Decision Trees}

A decision tree is a simple form of classification which involves a series, or "tree" of classifications, in which an unknown period of data is categorized into increasingly specific subsets based on threshold values of a particular feature. For instance, the first node of an activity classification decision tree may separate periods of human activity data into either a static or active subset based on the average magnitude of acceleration of the shank. If the mean acceleration magnitude is above a certain threshold value, the period will be classified as active; otherwise it will be classified as static.

Decision trees can be constructed either manually, based on human intuition or heuristic measures, or automatically by examining training data to determinine appropriate features for decision making. Popular automatic decision tree algorithms 
include $\mathrm{ID} 3^{42}, \mathrm{C} 4.5^{43}$, and $\mathrm{CART}^{44}$. Figure 3 shows the structure of a custom decision tree. Decision trees have several advantages. They are intuitive and simple to understand and perform well on large sets of data with high computational efficiency. The disadvantage is that manually constructed trees may not choose optimal decision nodes or thresholds, while automatically constructed trees may overcomplicate decisions and decrease computational efficiency or accuracy.

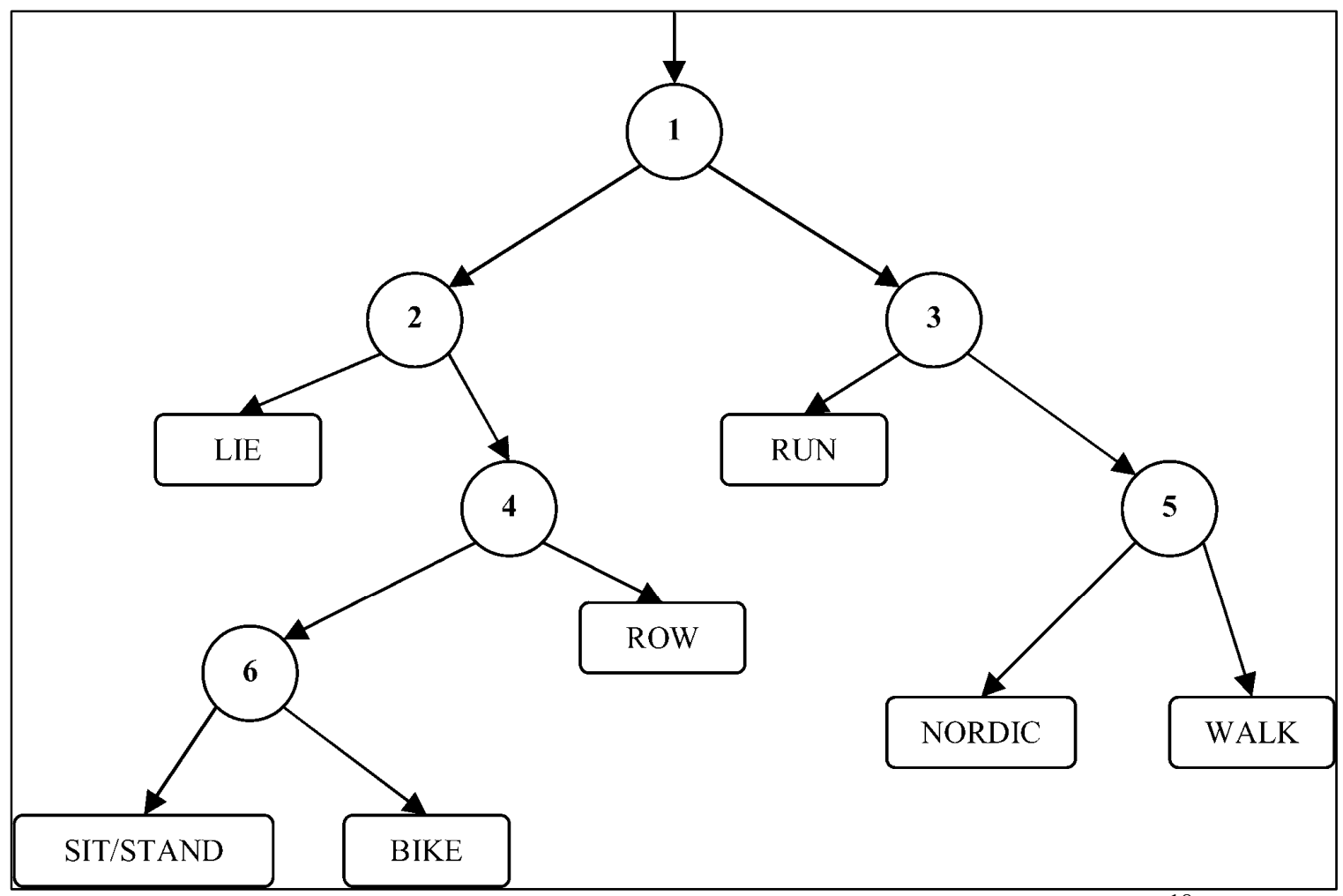

Figure 3. Example of a custom decision tree for activity classification ${ }^{18}$

Decision trees also tend to overclassify, that is, all periods of movement data introduced to the tree will be classified as one of the available decision subsets, even if the period of data does not resemble any of the final subsets. For instance, if the available subsets within a tree were all gait activities such as walking, running, and stair climbing, 
and a period of static posture such as sitting or lying were introduced to the tree, it must be classified as one of the gait activities. To get around this, many advanced decision tree algorithms include the option to create new decision "branches."

\subsubsection{Gaussian Mixture Models}

Gaussian mixture models (GMMs) are a form of classification using probability density functions which have gained popularity in speech recognition algorithms. These models assume that each observation, or feature, within a subset follows a Gaussian distribution. In activity classification, GMMs assign classifications by appropriately examining the features of an unknown period of activity, and determining for each training subset the probability that the unknown period falls within that subset. The unknown period is assigned to the subset for which it has the highest probability. GMMs have several advantages. The probability distributions provide accurate classification by differentiating distinct peaks between clusters, assuming the training clusters are "tight," and the semi-parametric nature allows gaps from sparse training data to be filled ${ }^{45}$. In addition, outliers can be easily identified. Unlike decision trees, if the probability for any subset does not meet a certain threshold, the unknown period can be defined simply as an outlier, or undefined activity. These models assume, however that observations follow Gaussian distributions. Features must be chosen carefully to ensure that this assumption is appropriate. In addition, GMMs are not as computationally efficient as decision trees, since a new probability density must be calculated for each data point. 


\subsubsection{Hidden Markov Models}

Like GMMs, Hidden Markov models (HMMs) were popularized by their use in speech recognition algorithms. HMMs are essentially a fusion of Markov chains and mixture models. HMMs are advanced mixture models; they make classifications based on the given "state" of observation probability distributions. Figure 4 demonstrates the structure of the HMM.

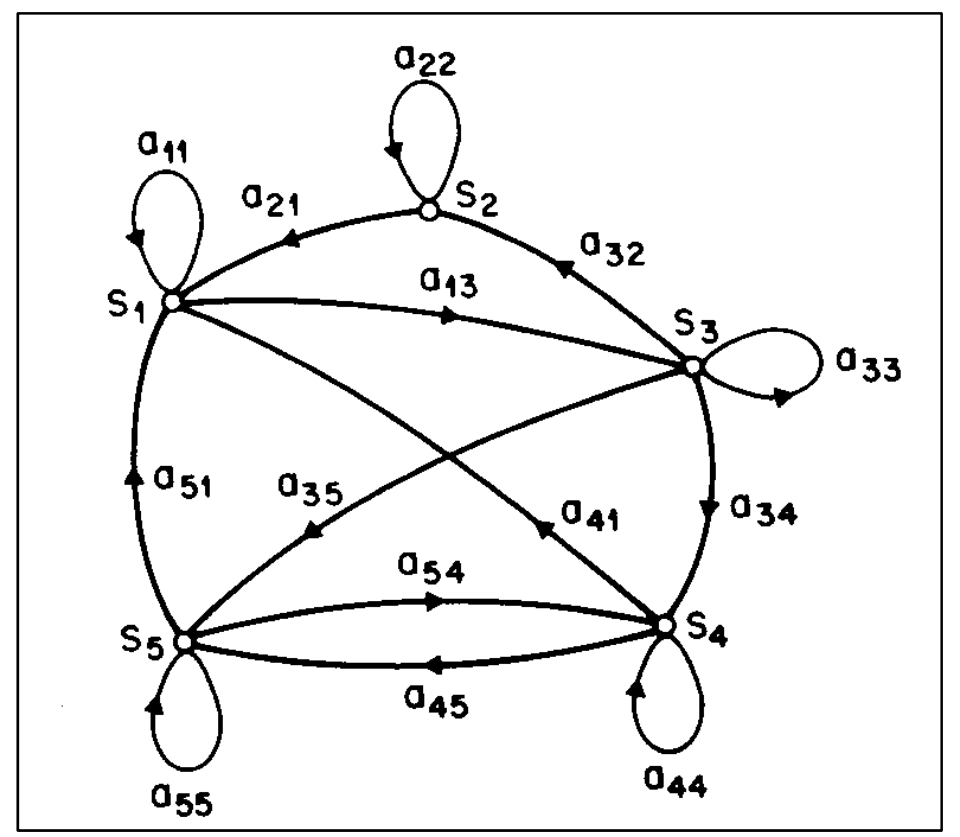

Figure 4. A Hidden Markov model of 5 states, with $\mathbf{a}_{\mathbf{i j}}$ representing transition probabilities between states ${ }^{46}$.

Whereas in GMMs there is only one "state," a Gaussian distribution, HMMs have multiple states, where for each new data point there may be a transition between states depending on the transition probability distribution between the states (the Markov chain $)^{47}$. In activity classification, HMMs provide a distinct advantage over GMMs. One of the problems with GMMs is that they do not incorporate transition probabilities 
between several states. For instance, if a subject is riding an elevator, it is highly likely that when the elevator stops and the doors open, the subject will begin walking. HMMs account for this transition probability. This helps to smooth continuous activity data by making reasonable assumptions about the subjects future states based on past states, or vice versa. The issue with HMMs is that they are particularly computationally expensive when compared to other routines, given that they require multiple probability densities depending on the state, or activity, as well as probability densities for transitioning between states. Increasing the number of identifiable activities will significantly increase the computational cost.

\subsection{4 k-Nearest Neighbor Models}

k-Nearest neighbor $(\mathrm{kNN})$ models provide a simple, intuitive machine learning technique for activity classification. A set of observations, or features, are selected to represent each period of activity. Periods of defined activity are used to train the model, and the features of the training data occupy a feature space. kNN models classify an unknown activity period by calculating its distance within the feature space from the known activity periods of the training data, and assigning it to the class of its " $\mathrm{k}$ " nearest neighbors. A value of $\mathrm{k}=1$ would use only the nearest neighbor, while a value of $\mathrm{k}=5$ would consider the 5 nearest neighbors. The distances may be calculated by a variety of metrics, including Euclidean, standardized Euclidean, and Chebychev distances. Figure 5 illustrates kNN classification.

kNN models can be highly accurate when compared to other routines ${ }^{14}$, and their biggest advantage is simplicity. Because they require a distance calculation for each point 
in the feature space however, they can be computationally expensive, especially as the amount of training data increases.

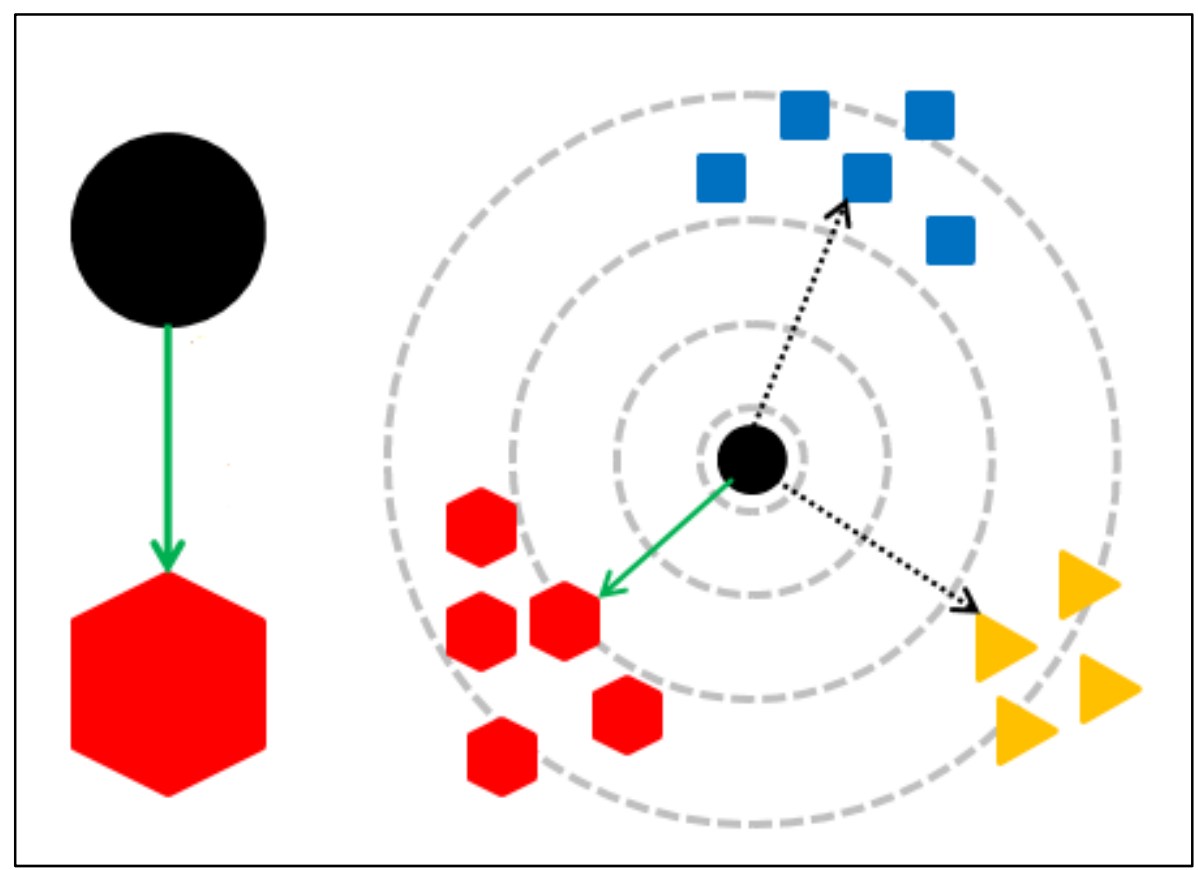

Figure $5 . \mathrm{A} \mathrm{k}=1 \mathrm{NN}$ model. The test data point is assigned to the class of its nearest neighbor.

\subsubsection{Artificial Neural Networks}

Artificial neural networks (ANNs) were developed to mathematically model the processes of the human nervous system. The human brain is highly capable of instantly recognizing types of activity, even with limited information. The ANNs behave by simulating the interconnection of neurons in the brain. ANNs consist of three neuron layers: input, hidden, and output neurons. They generally use a feed-forward approach, and connection between an artificial neuron is given a threshold value for "firing" that neuron. This value is determined from the model functions defining the relationships between each of the neurons. Figure 6 shows the basic decision structure of the ANN. 


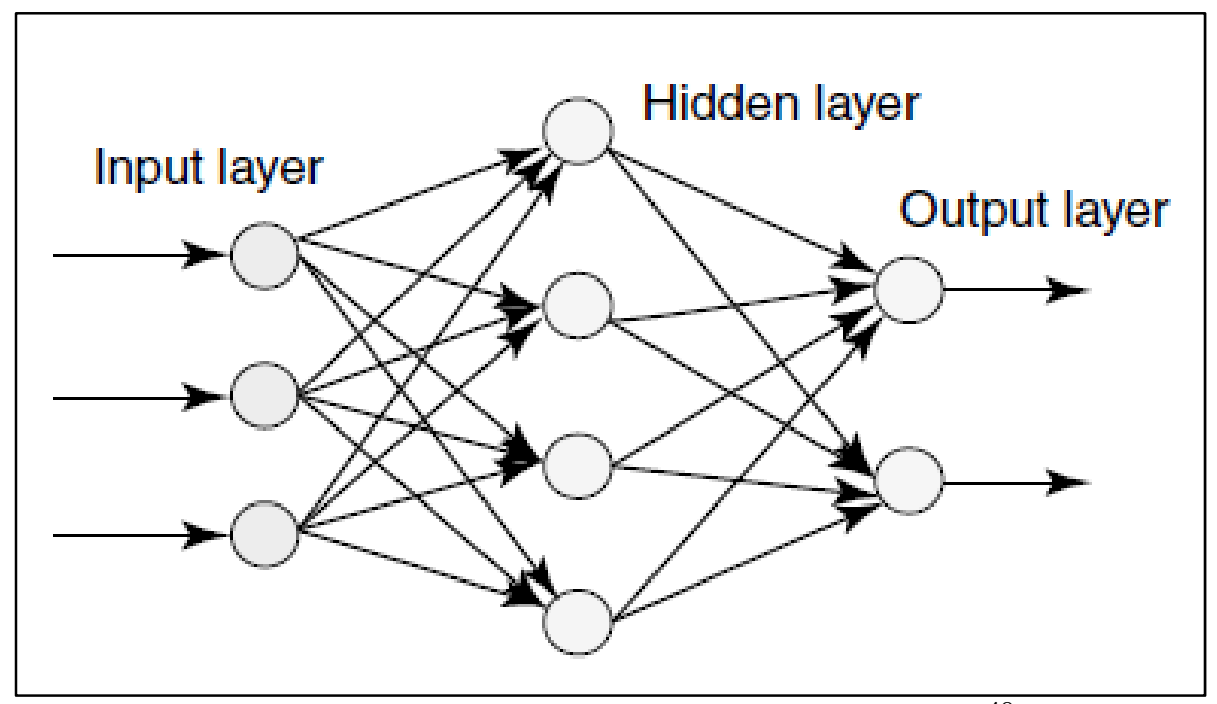

Figure 6. Multilayered artificial neural network ${ }^{48}$.

What makes ANNs attractive is their ability to engage in unsupervised learning. They seek the most efficient path between layers to maximize numerical reward. In the case of activity classification, this allows unsupervised use without previous definition of activity classes. The classes, however, must at some point be labeled in order to provide a descriptive classification.

\subsection{Selection of Data Mining Technique for This Work}

For this work, we used a hybrid technique consisting of a 6-level "decision" tree and kNN models for activity classification. When choosing from available models, we considered that the goals of this research were to provide a simple and efficient means which gave the most comprehensive view of subject activity with only shank and foot kinematic features, and to do so with minimal subject-specific training data. Figure 7 shows a schematic flowchart of the framework process. 


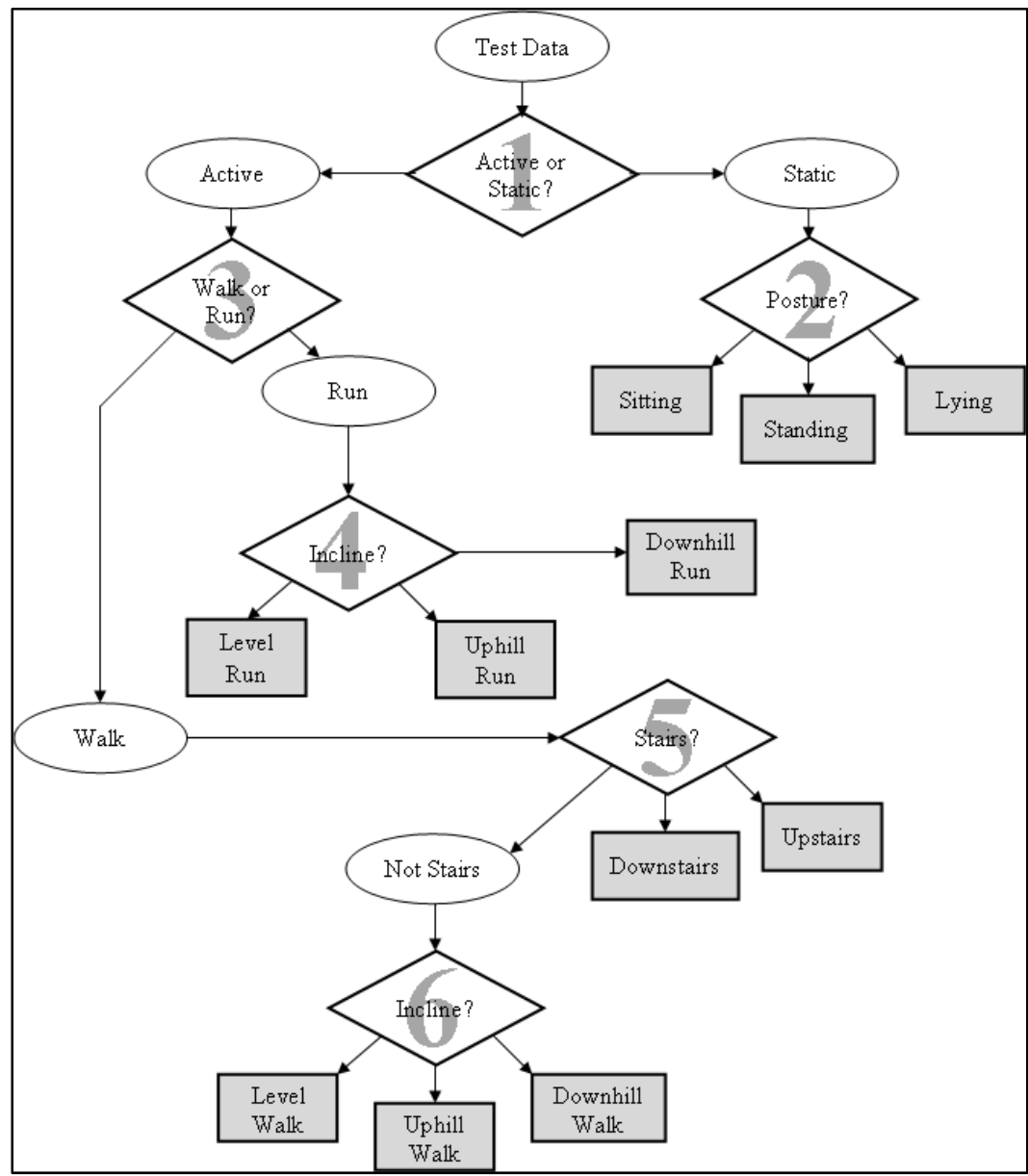

Figure 7. Classification Structure. Diamonds represent kNN routines, ovals represent intermediate classifications, and rectangles represent final classifications.

The framework was designed with kNN models for several reasons. The clearest advantage to using $\mathrm{kNN}$ models is their simplicity to implement and program. The bulk of the classification framework was developed in Matlab ${ }^{\circledR}$, and the software includes a statistic toolbox with $\mathrm{kNN}$ classifiers which are easy to modify and incorporate into a larger classification structure.

In this framework, the nearest neighbor classification replaced the traditional threshold based classifications at each level of the decision tree. This provided comprehensive classification while maintaining the tree structure. As discussed in Section 2.3.4, kNN models are intuitive and do not require complex training routines as 
those that may be required for GMMs, HMMs, or ANNs. Additionally, classification routines using $\mathrm{kNN}$ have been shown to be equally or more accurate than their more complex counterparts ${ }^{14,49}$.

The custom decision tree provides the advantage of classifying at several levels. For instance, at the first level, a period of data will be classified as active or static. Even with this data alone, a measure of activity level in the user can be determined. The routine could also classify periods of walking even if it is incapable of classifying the grade. If the framework consisted of only one level of kNN classification, no intermediate information would be available. By designing the framework with classification levels, we hoped to provide a picture of the user's activity even where complete classifications could not be made.

The goal of this research was not to develop a complex, novel data mining technique. Rather, we focused on implementing simple systems which allowed us to assess the efficacy of AFO-mounted TEMPO IMUs in activity classification. Nearest neighbor models within a decision tree structure provided the best combination of simplicity, adaptability, and accuracy. They allowed us to proficiently accomplish the goal of classifying activity using shank and foot kinematics from perturbed, AFO gait. 


\subsection{Activity Classification of Healthy Subjects Wearing Test AFOs}

The overarching purpose of this work is to assess the use of AFOs as a TEMPO sensor mounting platform for the means of classifying long-term activity in AFO users. This initial study examined healthy subjects wearing AFOs capable of perturbing their gait in conditions similar to those found in individuals with $\mathrm{CP}$. The primary goal of this initial study was to determine an accurate means for activity classification using only shank and foot kinematic data as would be available from AFO mounted sensors. The secondary goal of the study was to assess how perturbations in gait affect activity classification, and make a recommendation for future studies which will seek to classify activity in children with $\mathrm{CP}$ and assess spatio-temporal gait parameters using IMU data. This chapter examines the methods and results of this work, bearing in mind the aims of the study in assessing the efficacy of AFO-mounted IMUs for activity classification.

\subsection{Custom AFOs Modified to Induce Gait Perturbation}

This study used the modified AFOs designed for a previous study at the UVa Motion Analysis and Motor Performance Laboratory. For a complete description of this study, refer to "Center of Mass Motion and the Effects of Ankle Bracing on Metabolic Cost during Submaximal Walking Trials" by Herndon et al. ${ }^{50}$. This study examined the relationship between metabolic expenditures and vertical center of mass excursion in healthy adults who experienced perturbed gait via the use of the custom AFOs. 
The custom AFOs used in the Herndon study and the present study were designed in order to evaluate the reduction in ankle power as is caused by excessive plantarflexion or ankle stiffness. The AFOs were manufactured with a hinge at the ankle joint, which when uninhibited allowed up to $30^{\circ}$ rotation in either plantar- or dorsiflexion, enough to accommodate the normal range of motion during walking. The hinge was equipped with screws which could set the joint into a locked position at a neutral state, that is, a $90^{\circ}$ ankle angle which held the line of the shank orthogonal to the line of the foot. The AFO was also equipped with loops on the posterior of the AFO for mounting springs. To imitate equinus gait as found in many children with $\mathrm{CP}$, steel springs could be attached via the loops to connect the posterior of the shank with the posterior of the foot-plate, forcing the wearer into a state of plantarflexion and resisting dorsiflexion moments.

These AFOs enabled the researchers to assess both the primary and secondary goals of the study, serving as TEMPO mounting platforms and inducing perturbations of gait similar as those which may be found in individuals with CP. Indeed, Herndon et al. showed the AFO perturbations did affect the gait dynamics of the user, as shown in Figure 8. The results indicate significant increases in the metabolic cost and vertical center of mass displacement during walking at multiple speeds for the conditions when compared to walking wearing only shoes, with the most significant increases being found in the equinus gait condition ${ }^{50}$. The increases in vertical center of mass displacement and decrease in early peak ankle moment found by Herndon et al. for the equinus condition mirror results from past experiments examining those with actual pathological equinus gait from CP pathology ${ }^{51}$. 


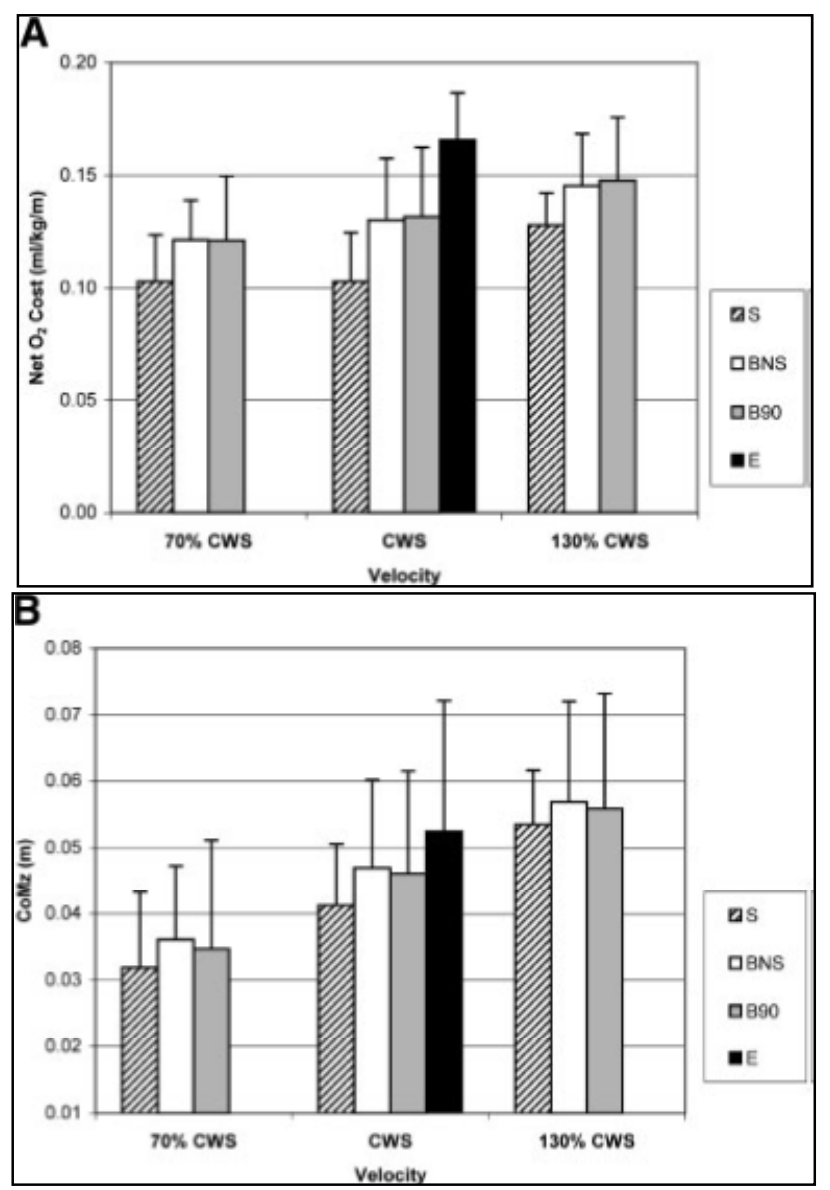

Figure 8. Results of Herndon et al. (A) shows an increases in net $\mathrm{O}_{2}$ cost of braced and perturbed walking, while (B) shows increases in vertical center of mass excursion.

\subsection{Activity Test Protocol}

For the initial study, eight (8) healthy, college-aged student volunteers ( 7 male, 1 female, weight $165.4 \pm 11.8 \mathrm{lbs}$, height $71.4 \pm 2.5 \mathrm{in}$ ) were recruited to participate in a data collection session to acquire shank and foot kinematic data for a set of gait activities and postures. Each of the subjects was fitted with the test AFOs described above and carried out an activity protocol under the three induced conditions: Free, Locked and Equinus. Free allowed free rotation of the ankle hinge up to $\pm 30^{\circ}$, Locked set the ankle joint at the neutral position, and Equinus used the posterior spring attachments to force 
the ankle into $30^{\circ}$ plantarflexion and resist dorsiflexion moments. Using tight-fitting Velcro®, TEMPO 3.1 sensors were attached to the lateral side of each AFO, about $10 \mathrm{~cm}$ inferior to the knee, and to the top of each shoe, about $15 \mathrm{~cm}$ anterior to the malleoli. The sensors were aligned on the shank such that the X-plane roughly corresponded to the frontal plane of the body, the Y-plane to the transverse plane, and the Z-plane to the sagittal plane. The sensors were aligned on the foot so that the X-plane roughly corresponded to the sagittal plane, the Y-plane to the frontal plane, and the Z-plane to the transverse plane. Subjects were also fitted with a set of fifteen lower body reflective markers to allow the collection of kinematic motion data via a Vicon ${ }^{\circledR}$ 8-camera motion capture system. Figure 9 pictures a subject wearing the AFOs with TEMPO 3.1 sensors and Vicon ${ }^{\circledR}$ markers attached, and the necessary hardware placed to induce the gait perturbations. Video and Vicon ${ }^{\circledR}$ data were used to create ground truth descriptions of the activity collection protocols as well as validate feature reduction from kinematic data collected via the TEMPO 3.1 sensors.

Under each gait condition, the subjects performed, in pseudo-random order, the following activities: walking $(4 \mathrm{~km} / \mathrm{hr})$ and running $(7 \mathrm{~km} / \mathrm{hr})$ on a Biodex ${ }^{\circledR}$ treadmill at a level grade $(0 \%)$, decline $(-15 \%)$, and incline $(15 \%)$, overground level walking, walking up and down a flight of four wooden stairs, sitting on a stool, standing, and lying down on a foam pad. During the activity protocols, the researchers collected video from two cameras set orthogonally to view the entire lab space. All subjects signed a University of Virginia IRB approved consent form. 


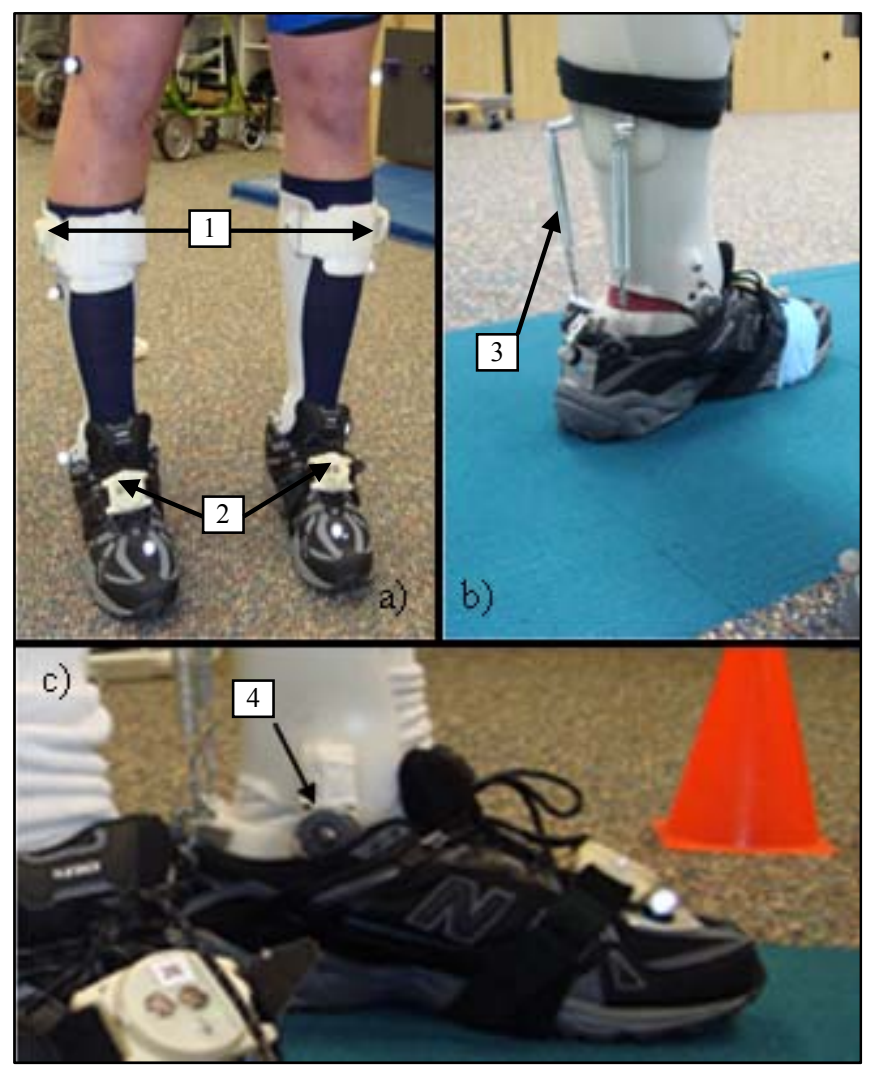

Figure 9. Test AFOs a) [1] and [2] indicate the shank and foot mounted TEMPO sensors, respectively. b) [3] indicates the shank-foot spring arrangement. c) 4 indicates the ankle hinge, which could be locked via the two small screws seen just above the hinge

Kinematic data from the TEMPO 3.1 accelerometers and gyroscopes was collected at $128 \mathrm{~Hz}$ on a Bluetooth-equipped PC and annotated using a graphic interface developed in Labview, while kinematic Vicon ${ }^{\circledR}$ data and video were collected at $60 \mathrm{~Hz}$ using Vicon ${ }^{\circledR}$ Workstation software. Figure 10 presents a sample of the TEMPO 3.1 data from the shank and foot during a transition from walking to running. Each period of defined activity or posture was annotated with start and stop timestamps in order to synchronize TEMPO 3.1 data with Vicon ${ }^{\circledR}$ and video data. Timestamps provided a means of developing ground truth descriptions throughout the entirety of each trial. In between annotated periods, the subject moved freely within the laboratory while researchers prepared equipment for the next activity (treadmill, stairs, etc). 

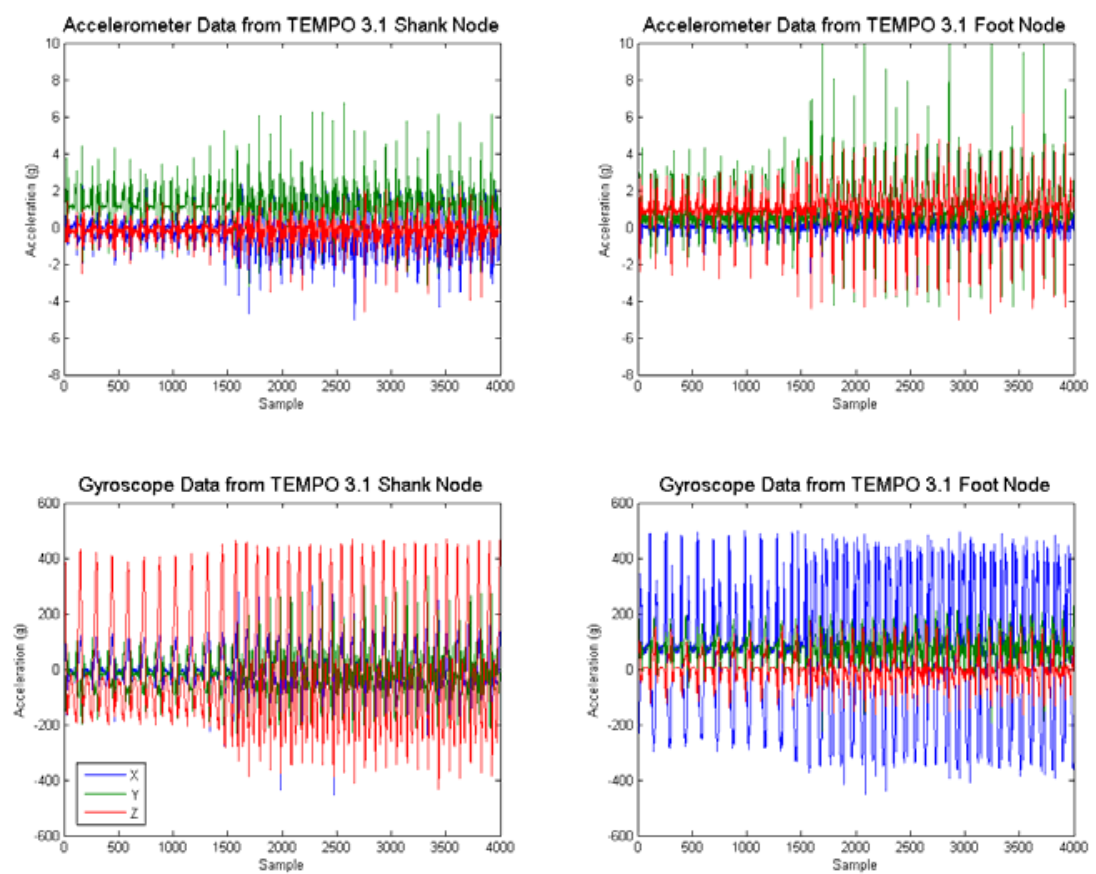

Figure 10. Sample Data from Walking-Running Transition. Axis labels represent sensor axes as shown in Figure 2.

For five of the subjects, video was also collected continuously throughout each trial. This gave the ability to test the algorithm's sensitivity when the subject moved about freely, demonstrating framework performance when analyzing semi-natural movement of greater than a few minutes. In the description of this thesis work, all data windows which contain predefined activity will be termed "annotated," while all data windows which contain semi-natural activity outside of the annotated periods will be termed "non-annotated." All three AFO conditions from seven subjects were tested, excluding the Equinus condition for two subjects, giving a total of 19 different gait presentations. The data from the eighth subject and the Equinus from two subjects were unusable due to Bluetooth miscommunication and/or TEMPO 3.1 malfunction. The 
average time of annotated activity for each trial was 4 minutes and 42 seconds. The average total duration of each trial was 24 minutes.

\subsection{Signal Processing and Data Reduction}

Before classifications could be made, raw data signals from the TEMPO nodes were processed so a relevant set of data features could be reduced. Trial data was divided into discrete data windows, and for each window a feature vector was compiled to enable activity classification.

\subsubsection{Data Windowing}

To enable feature reduction, data streams from the TEMPO 3.1 sensors were partitioned into 2 -second windows, with 1 second of overlap between windows. The window size of 2 seconds was sufficient for capturing a complete gait cycle in the healthy subjects of this study. Waters et al. has demonstrated that healthy humans walk with a cadence of approximately between 100 and 120 steps per minute ${ }^{52}$. Since a gait cycle consists of a complete stride, or two steps, this corresponds to between $1.0-1.2$ seconds per gait cycle. Future studies will examine how atypical or pathological gait patterns necessitate a change in window size for the capture of a complete gait cycle, though studies have indicated that cerebral palsy and AFO use do not significantly lessen cadence $^{53}$. Damiano and Abel demonstrated that gross motor function (GMFM) scores in children with $\mathrm{CP}$ are highly correlated with cadence, and that even those with severe GMFM limitation exhibited a cadence of more than $80 \mathrm{steps} / \mathrm{min}^{54}$. Regardless, the 
window size can be adjusted on an individual basis in order to capture the gait cycle of the specific AFO user.

Data windows allow the extraction of discrete time-independent features of the gait cycle such as maximum shank angles or frequency domain features such as fast Fourier transform (FFT) coefficients. The window size and overlap provide one window for every second of data, and an average of 1,426 windows per trial, of which an average of 282 contained annotated activity. Each sensor data window consisted of six 256sample data column vectors representing the three orthogonal axes of both accelerometer and gyroscope signal.

\subsubsection{Kinematic Feature Reduction}

Nearest neighbor classification models assign data windows to the class of their nearest neighbor window, chosen by the minimal distance within the feature space. A set of $n$ kinematic features populate the $n$-dimensional feature space, and a feature vector for each window provides a kinematic "snapshot" of the activity data. For this study, a set of 23 features were reduced from the accelerometer and gyroscope data, listed in Table 1.

Table 1. List of feature descriptions and number of degrees of freedom (DOF) for each window of data.

\begin{tabular}{lc}
\hline Feature Description & DOF \\
\hline Standard deviation (SD) of the acceleration magnitude for both Shank and Foot & $\mathbf{2}$ \\
SD of the tri-axial components of acceleration for both Shank and Foot & $\mathbf{6}$ \\
Gravitational orientation components for both Shank and Foot & $\mathbf{6}$ \\
Maximum rotational velocity components for both Shank and Foot & $\mathbf{6}$ \\
Maximum angle of the Shank in the sagittal plane & $\mathbf{1}$ \\
Maximum + Minimum translational velocity of the foot in the vertical direction & $\mathbf{1}$ \\
Fast Fourier transform (FFT) coefficient of vertical foot acceleration & $\mathbf{1}$ \\
\hline
\end{tabular}


The selection of features has a significant effect on the accuracy of the classification routine; selecting too few features or features which are indistinct between events will obstruct accurate classification. Conversely, selecting more features than is necessary for classification will increase computational costs and may lead to feature confusion. The list of features for this study was compiled based on results from previous work in IMU activity classification, as well as heuristically based on an understanding of gait patterns and human movement analysis. The justification for the inclusion of each feature is indicated below.

\section{Standard Deviations of Acceleration Components}

The standard deviations of the acceleration components over the 2-second data window have been found to be one of the simplest metrics for differentiating static posture from activity ${ }^{55}$. Figure 11 shows standard deviations for acceleration signals during walking and during standing.

At rest, the raw accelerometer output magnitude will equal approximately $1 \mathrm{~g}(9.8$ $\mathrm{m} / \mathrm{s}^{2}$ ) in the vertical direction, accounting for the force of gravity. The low frequency gravitational component of the acceleration can be suppressed with high-pass filtering, providing a filtered acceleration of $0 \mathrm{~g}$ during completely static activities. While overall acceleration magnitude can provide a reasonable differentiation between periods of activity and posture, standard deviations of the acceleration provide a description of acceleration change magnitude while maintaining robustness to outliers in a window, such as a twitch during a period of standing. This feature was acquired by calculating the standard deviation of each axis component of filtered accelerometer data as well as for 
the magnitude of the acceleration for both the shank and foot sensors. The accelerometer signal was band pass filtered with a bidirectional $3^{\text {rd }}$ order Butterworth filter between 0.3 $\mathrm{Hz}$ and $17 \mathrm{~Hz}$. This filtering process suppressed the low-frequency gravitational components of the accelerometer signal and the high frequency noise characteristics, while passing signals within the frequencies of human motion. Several other accelerometry studies have indicated that these frequencies are appropriate for human motion analysis ${ }^{17,19,35}$.
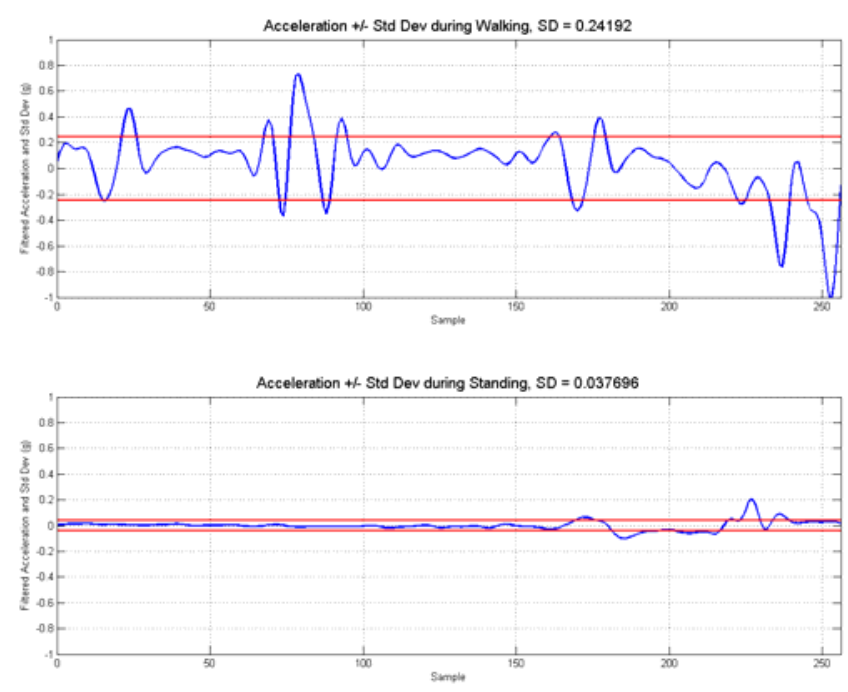

Figure 11. Standard deviations of the acceleration signal during walking (top) and standing (bottom). Note the slight twitch at the end of the standing window.

\section{Gravitational Orientation Components}

The gravitational orientation components of the acceleration signal provide a low frequency definition of the orientation of the IMU and the body segment to which it is attached. These features are reliable means of differentiating static postures via changes in shank and foot position. They are less applicable for cyclic gait activities since they 
sample at a frequency much less than that of a typical gait cadence. For static posture differentiation, however, they are more reliable than angles derived from filtered gyroscope signal integration, due to integration errors caused by the presence of noise and drift in the gyroscopes signal. Figure 12 shows the change in the gravitational orientation components during a transition from standing to lying.

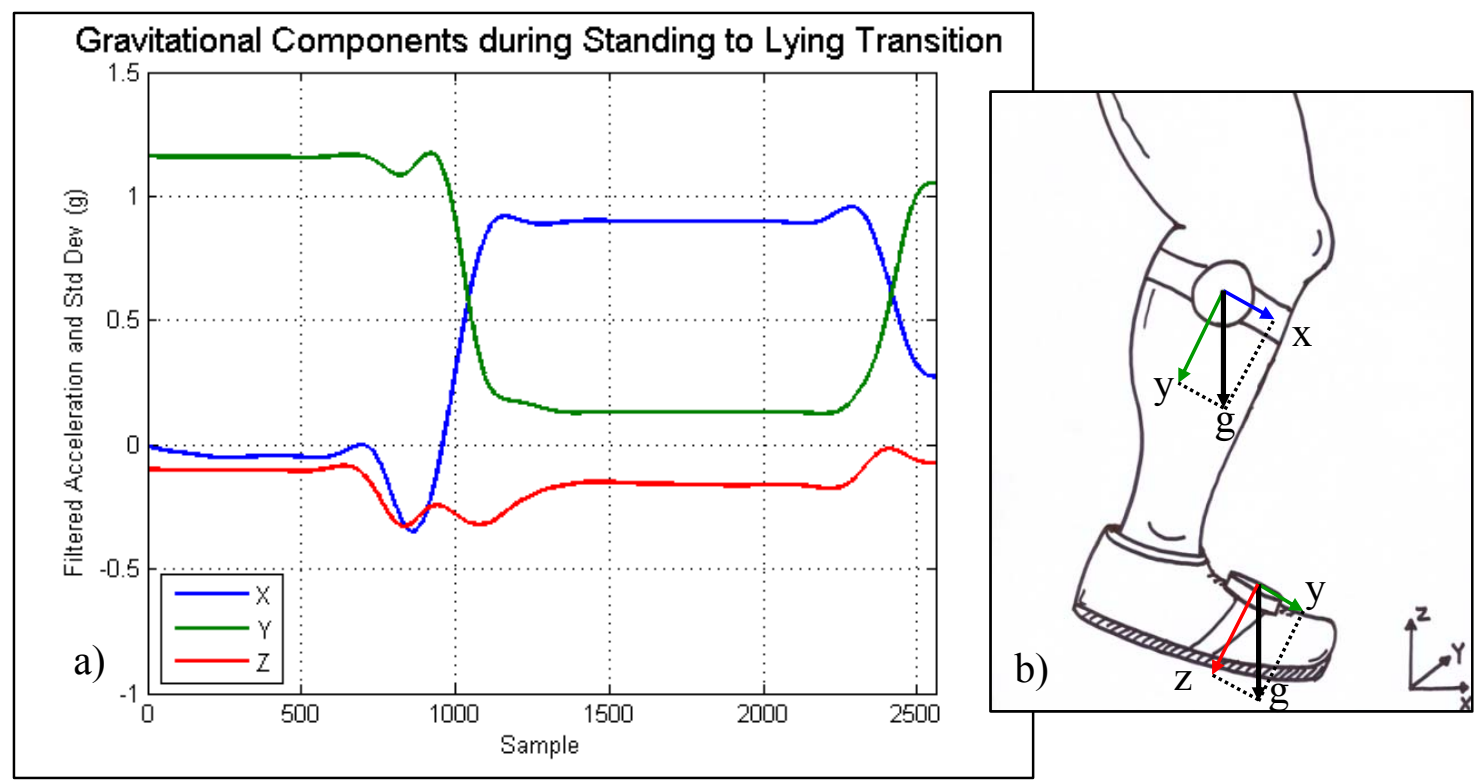

Figure 12. a) Gravitational Orientation Components of the SHANK node during transition from an upright standing position to a supine lying position. b) Diagram of gravitational components with mounted sensors.

The gravitational orientation components were calculated by applying a low pass $3^{\text {rd }}$ order bidirectional Butterworth filter with a cutoff frequency of $0.5 \mathrm{~Hz}$ to the accelerometer signal. This suppressed all but the gravitational component of the accelerometer signal to provide a measure of the orientation of the IMU. The low pass filter cutoff also reduced errors from high frequency noise characteristics of the accelerometer signal. 


\section{Maximum Rotational Velocity Components}

The maximum rotational velocity components can be used to differentiate gait events at varied inclines. They have specifically been useful in recognizing stair climbing events $^{56}$. They were calculated by finding the maximum rotational velocity of each component $(\mathrm{X}, \mathrm{Y}$, and $\mathrm{Z})$ of the filtered gyroscope signal in each 2-second data window. A $3^{\text {rd }}$ order bidirectional Butterworth high pass filter with a cutoff frequency of $0.3 \mathrm{~Hz}$ is used to suppress errors caused by low frequency sensor drift. Figure 13 pictures the maximum rotational velocity components of the shank for various gait activities, showing distinctions in the shank rotational velocity components between walking and running events as well as for stair use.

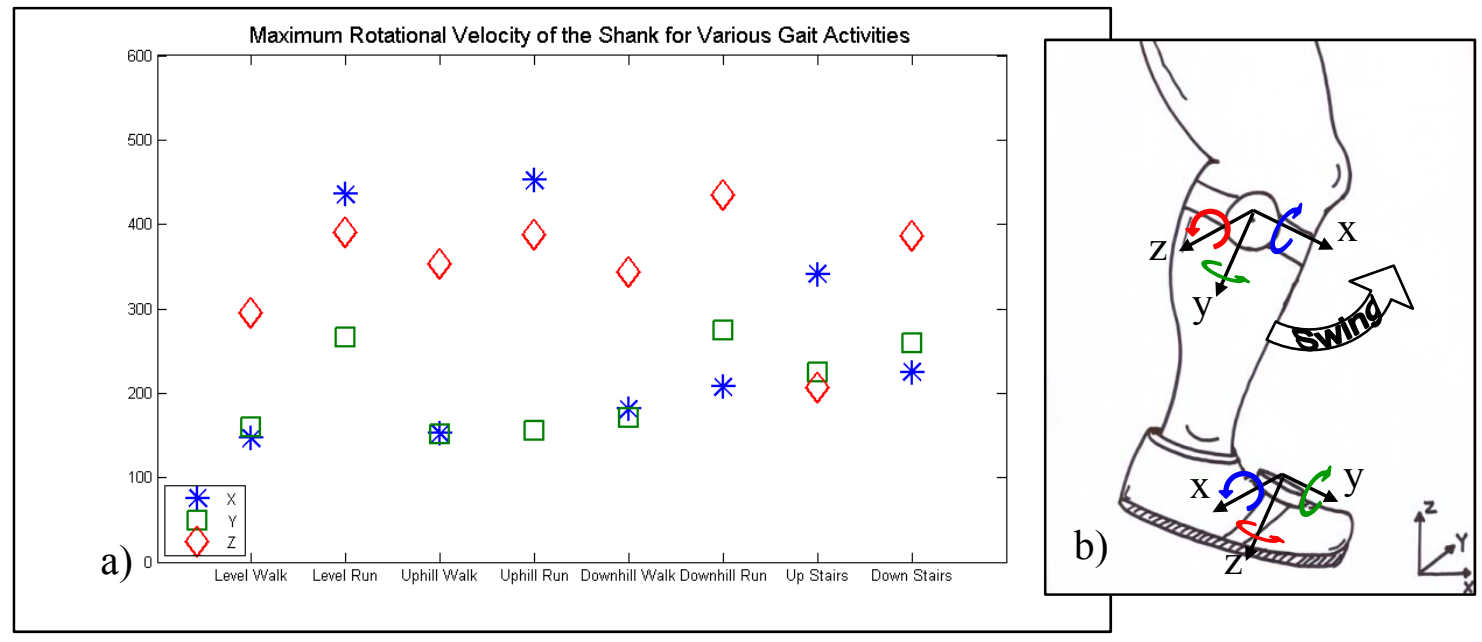

Figure 13. a) Maximum rotational velocity components for various gait activities.

b) Diagram of rotational velocities during leg swing.

\section{Maximum Angle of the Shank in the Sagittal Plane}

The maximum angle of the shank in the sagittal plane can provide a distinction between inclines during gait activities, as well as stair use. The angles were calculated by 
integrating the gyroscope signal, high pass filtered at $0.3 \mathrm{~Hz}$ to remove the low frequency drift errors associated with gyroscope sensors. Figure 14 shows the maximum shank angles in the sagittal plane for various gait activities.

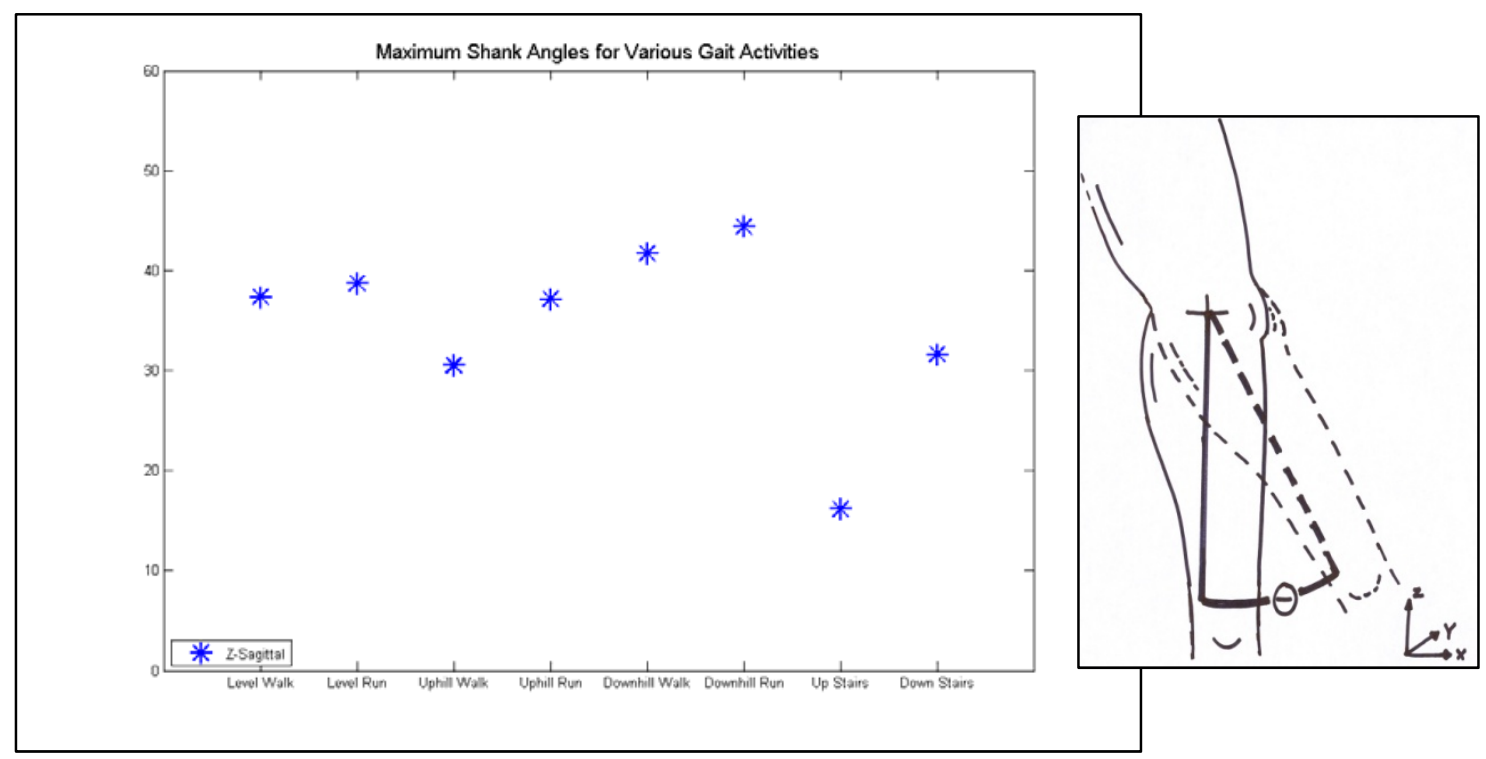

Figure 14. Maximum shank angle, $\theta$, in the sagittal plane for different activities. The results show some separation for incline gait activities, and a large separation for stair climbing.

The integration was performed numerically in the manner described by Chen et al.:

$$
\varphi(n)=\varphi(n-1)+\Delta / 2 *[\omega(n-1)+\omega(n)]
$$

where $\varphi$ is the angle, $\Delta$ is the sampling period (1/128 s), and $\omega$ is the rotational velocity from the filtered gyroscope signal ${ }^{35}$. This method was validated using Vicon optical data to ensure that the filtering process to remove drift error did not adversely affect the angle integration. The TEMPO ankle angle was calculated as the difference between the integrated sagittal plane shank (Z-axis) and foot (X-axis) filtered gyroscope signals. The TEMPO ankle angle was compared to the Vicon ${ }^{\circledR}$ data collected during a period of level 
treadmill walking, as shown in Figure 15. The RMSE for the two angles measurements was 2.547 degrees, which is within the error margin of the Vicon ${ }^{\circledR}$ reference system $(\sim 3$ degrees).

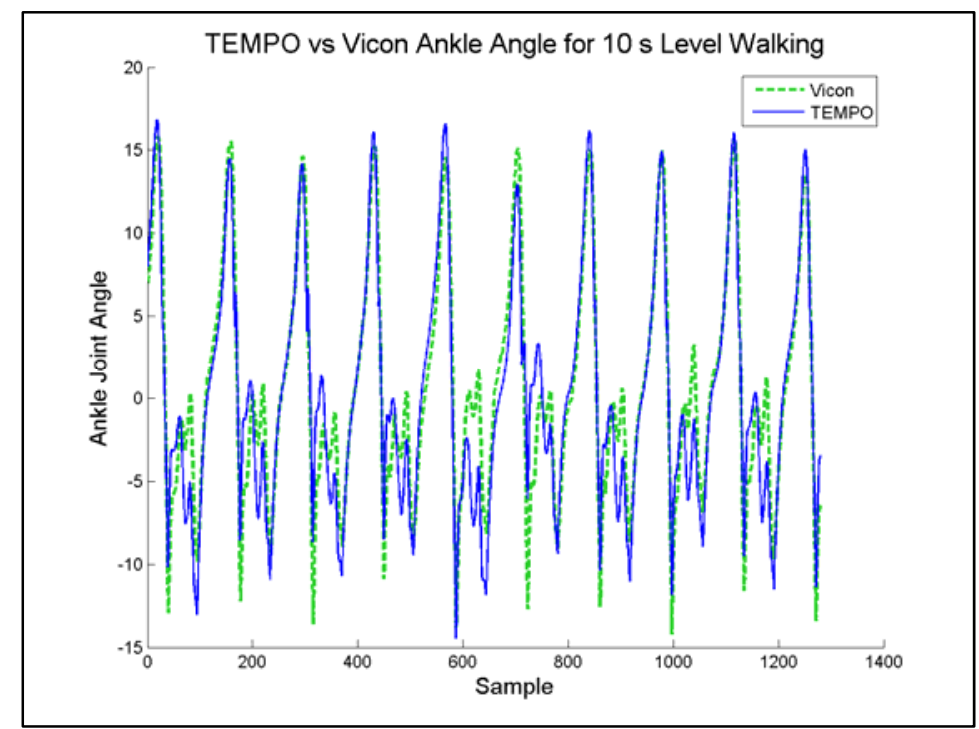

Figure 15. Ankle angle during walking. Solid blue line is TEMPO ankle angle and dotted green line is $\mathrm{Vicon}{ }^{\circledR}$ reference system ankle angle. Maximum values from TEMPO are largely consistent with those from Vicon ${ }^{\circledR}$. RMSE $=2.547$ degrees

The angle computation is dependent on the value chosen as the initial angle. For the activity protocol tested, the data collection always began when the subject was standing, and defining a zero angle at this point provided some cross-subject consistency. Future studies which consider cross subject training or classification of natural movement must take care to define the initial angle, or correct the angle at a point of reference. This can be done easily if a period of standing can be identified.

\section{Sum of the Maximum and Minimum Vertical Foot Velocities}

The sum of the maximum and minimum values of the vertical foot velocity was determined heuristically to be a useful metric for differentiating stair ascent and descent. 
During gait, the vertical velocity of the foot alternates between positive and negative values as the foot is raised and lowered. It was often found that during stair descent the sum of the maximum and minimum velocities was negative, while during ascent this value was positive or near zero. A chart of this feature for stair ascent and descent is provided in Figure 16.

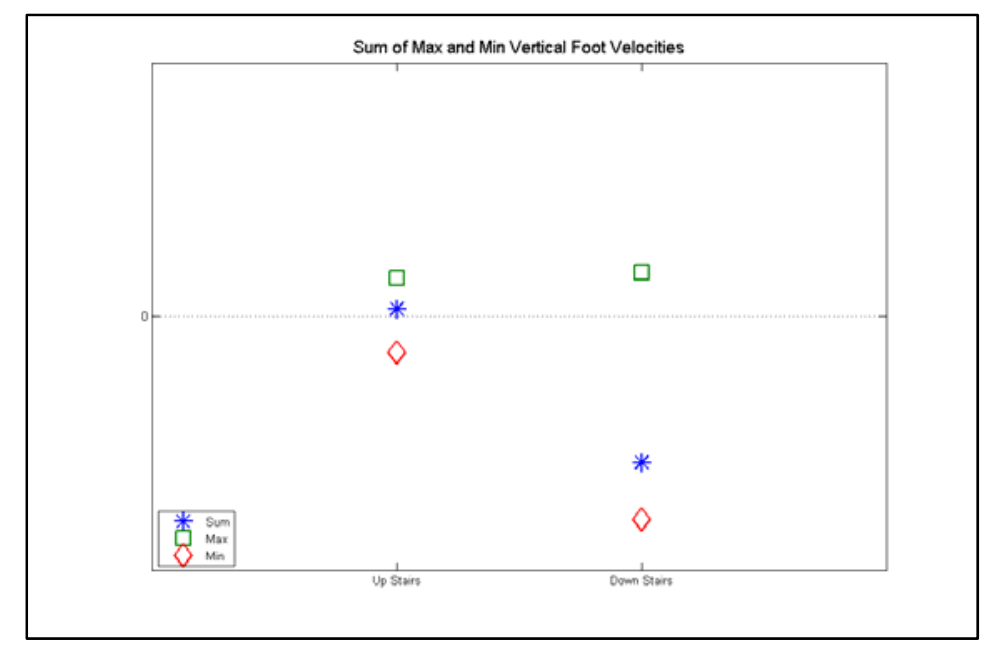

Figure 16. Sum of maximum and minimum foot velocities during stair ascent and descent.

Vertical foot velocity was calculated by integrating the vertical $z$ component of band pass filtered accelerometer signal from the foot node over each window assuming zero initial velocity in the same manner as demonstrated above for the angle integrations:

$$
v_{z}(n)=v_{z}(n-1)+\Delta / 2 *\left[a_{z}(n-1)+a_{z}(n)\right]
$$

where $v_{z}$ is the vertical velocity, $\Delta$ is the sampling period $(1 / 128 \mathrm{~s})$, and $a_{z}$ is the vertical foot acceleration. Moments of foot contact were then located by indexing periods where foot accelerations and changes in acceleration were at or near zero, a threshold defined by 
a quarter of the standard deviation of the acceleration signal for the data window. Figure 17 provides a graphical interpretation of this process.

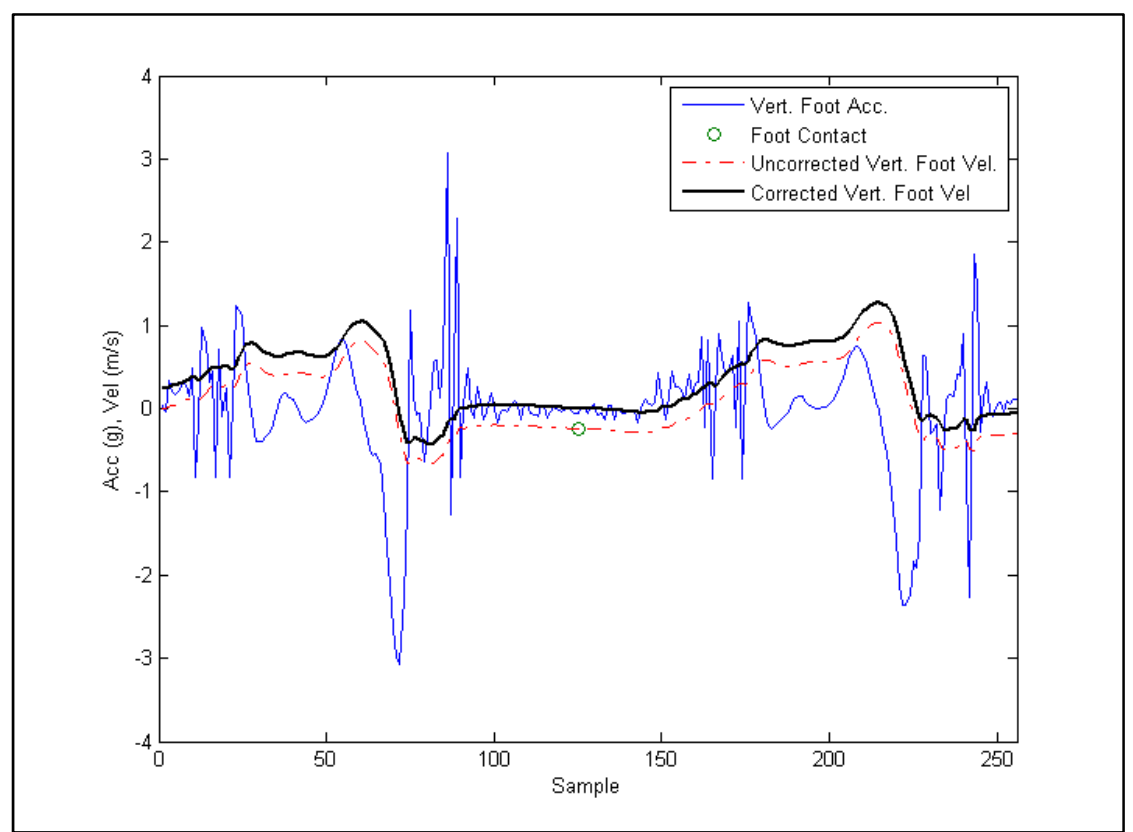

Figure 17. Velocity correction process. The routine integrates the acceleration signal and then corrects the velocity to zero at the midpoint of foot contact.

\section{Fast Fourier Transform Coefficients for Vertical Foot Acceleration}

The FFT coefficients from kinematic sensors have been shown to provide a distinction between rate-dependent cyclic events such as walking and running ${ }^{14,57}$. For this study, the FFT coefficients of the vertical foot acceleration were used as an indicator of gait cadence. To acquire the coefficients, the Z-axis acceleration signal of the foot node was low-pass filtered with a $3^{\text {rd }}$ order bidirectional Butterworth filter at a cutoff frequency of $17 \mathrm{~Hz}$. The discrete Fourier transform (DFT) of the filtered signal was computed, and the frequency associated with the highest response coefficient was returned. 


\subsubsection{Notes on the Feature Set}

The feature space provided a kinematic view of the subjects' activity during each trial. For perturbed conditions, we found that the variability within the features generally increased. The standard deviations for the training feature sets of Equinus and Locked conditions increased by an average of $33 \%$ and $24 \%$, respectively, over those of the Free condition. This presentation of gait variability in the study feature space was consistent with the results of other studies which examined variability in gait of AFO users and those with $\mathrm{CP}^{7,49,50}$.

It should be noted that all 23 features were calculated for each data window regardless of the presence of a gait activity. As such, certain features only provided meaningful distinctions when certain activities or postures were actually present. For instance, FFT coefficients are irrelevant when differentiating between static postures. Feature selection methods based on significance within subclassifications will be discussed in Section 3.6.

It should also be noted that integrating the signal of either a gyroscope or accelerometer is prone to error. Non-filtered signals retain low-frequency drift and gravitational components which can cause significant accumulation of error even over relatively short periods of data. Filtering to remove or suppress low-frequency sources of error strips the signal of some descriptive kinematic data, even if the cutoff frequency and roll off of the filter are selected carefully. Often times special techniques such as Kalman filtering and quaternion sensor fusion are employed to overcome these errors ${ }^{58-60}$. For this study, however, high levels of accuracy were not required in integration calculations. Activity classification relies on distinction between features; the relative values are more 
important than the absolute magnitudes. Band pass filtering the IMU signals provided accelerations and rotational velocities with suppressed low frequency error and high frequency noise, making integration without error inflation feasible. Over short data periods $(\sim 2 \mathrm{~s})$, integration errors remained small, and permit the level of accuracy required for this study.

\subsection{Data Training and Distance Calculation}

Data was partitioned from each trial to train the classification algorithm on each activity and posture. For nearest neighbor classifications, the distances from test data windows to training data windows within the feature space were calculated. This section describes the training data partitioning process and the distance method used for classification.

\subsubsection{Partitioning Training Data}

One of the goals of this study was to evaluate how training data size affects accuracy in nearest neighbor classifications. Collecting training data can be time consuming, and for the purposes of this research must be done within a laboratory setting. The computational cost of nearest neighbor classification routines is proportional to the size of the training data; the distance between the test data windows and each training data window must be calculated. Since the future of this research is aimed at monitoring gait and classifying activity in natural settings during everyday activity, it will be 
important to understand the effects of relative training data size on classification accuracy.

For training, a percentage of data was removed from the full trial data set, dividing the data into a training data set and test data set. Only annotated data was considered for the purposes of training, since one of the aims of this study is to examine classification of semi-natural activity using predefined activity for training. The data was selected such that for each level of training data selection, the same percentage of training data was partitioned for each annotated activity. For example, at a training data selection level of $50 \%$, if 20 annotated windows were collected for level walking and 8 for standing, 10 training windows would be selected for walking and 4 for standing. At least one window for each activity and posture was included in the training data.

Training data selection was randomized to reduce bias present in different stages of annotated activity. For instance, during stair climbing, the first step onto the stairs and the last step at the top will look kinematically different than steps while on the stairs. When training data was partitioned, a sample was chosen to incorporate data windows from random stages of the annotated activity. When results were assessed, twenty iterations were performed, each with a different random training set. The mean sensitivities and their standard deviations were observed.

\subsubsection{Nearest Neighbor Distance Calculation}

At each of the six tree levels, a nearest neighbor classification was performed. The training data was represented in an $w_{r}-b y-m$ matrix $\boldsymbol{X}$ and the test data in a $w_{s}-b y-m$ matrix $\boldsymbol{Y}$, where $w_{r}$ and $w_{s}$ were the number of training windows and test windows, 
respectively, and $m$ was the number of features calculated, 23 in the case of this study. The coordinate distance, $d$, between a test window (rows in $\boldsymbol{X}$ ) and a training window (rows in $\mathbf{Y}$ ) was weighted by the square of the standard deviation (variance) of each feature column in the training data (columns in $\boldsymbol{X}$ ). That is,

$$
d_{i}{ }^{\prime}=\frac{\left(x_{i}-y_{i}\right)}{\boldsymbol{S}(i)}
$$

where $d_{i}{ }^{\prime}$ is the standardized distance, $x_{i}$ is the scalar value of the $i^{\text {th }}$ feature in the training data window, $y_{i}$ is the scalar value of the $i^{\text {th }}$ feature in the test data window, and $\boldsymbol{S}$ is the vector of the variance of the each column in $\boldsymbol{X}$, defined by

$$
\boldsymbol{S}=\left[\sigma\left(\boldsymbol{X}_{\boldsymbol{i}}\right)\right]^{2}
$$

where $\boldsymbol{X}_{\boldsymbol{i}}$ is the column vector containing the scalar values of the $i^{\text {th }}$ feature for the entire training data set and $\sigma$ is its standard deviation. By this process, feature vectors with large variance were given less weight than feature vectors with small variance. The distance, $\mathrm{D}$, between the test window and training window was then calculated by:

$$
D=\sqrt{\sum_{i=1}^{m}\left(d_{i}{ }^{\prime}\right)^{2}}
$$

A total of $w_{r}$ distances between each test window and the training windows were calculated. The test window was classified to the group of the training window for which the minimal distance was found. 
The weighting process minimized the effect of feature magnitude on variance importance. Specifically, the variances of those features with larger magnitudes were not considered more important than those of the features with smaller magnitudes. Without standardization, features with larger absolute magnitude (e.g. rotational velocities, angles) would have more weight than those with small magnitude (e.g. standard deviations, orientation components), as their variances would be intrinsically larger. Other distance metrics were considered, specifically: Euclidean, Chebychev, correlation, cosine, and city block distances. Table 2 shows the average sensitivity results using each distance metric.

Table 2. Mean Sensitivities for various nearest neighbor distance metrics

\begin{tabular}{c|cccccc}
\hline $\begin{array}{c}\text { Distance } \\
\text { Metric }\end{array}$ & Euclidean & $\begin{array}{c}\text { Standardized } \\
\text { Euclidean }\end{array}$ & Chebychev & Correlation & Cosine & $\begin{array}{c}\text { City } \\
\text { Block }\end{array}$ \\
\hline $\begin{array}{c}\text { Mean } \\
\text { Sensitivity } \\
(\%)\end{array}$ & 91.4 & 94.2 & 90.2 & 88.6 & 88.6 & 92.2 \\
\hline
\end{tabular}

The standardized Euclidean distance clearly provided the best sensitivity results for the study.

\subsection{Results for Forced Classification}

For this study, a value of $\mathrm{k}=1$ was chosen for the nearest neighbor classifications. This decision was made for several reasons, the key factor being a limited amount of training data. This will be discussed in more detail in Section 3.5.2. The initial assessment of the framework performance used forced classification, that is, all data 
windows were classified as one of the eleven predefined activities. For the purpose of this preliminary evaluation, results are expressed in terms of sensitivity, defined as

$$
\text { Sensitivity }=\frac{T P}{T P+F N}
$$

where TP is the number of true positives and $\mathrm{FN}$ is the number of false negatives. Since classifications were forced into one of the predefined activity or posture groups, performance metrics which consider false positives, such as specificity or positive predictive value, were not calculated. Specificity will be introduced in Section 3.6, where a distance-based declassification threshold will be considered.

When examining the classification of annotated data, the results for all activities and postures were included. For non-annotated results, only walking and standing were considered in the results. This is due to the fact that no subject engaged any other of the defined activities or postures for a significant amount of time ( $>2$ seconds) during nonannotated periods, even though they were not constrained by the researchers to do so. This fact illustrates a key distinction between semi-natural and natural movement; even when subjects are not physically or verbally restrained from certain activities, unfamiliar clinical settings may deter normal subject behavior.

\subsubsection{Sensitivity as a Function of Training Data Size}

To assess the effects of training data size, the classification scheme was trained using a variable-sized training data set. The researchers specified a percentage of annotated data to be used for training, ranging from $5 \%$ to $90 \%$ in $5 \%$ increments. This corresponded to $\sim 15$ seconds (5\%) to $\sim 250$ seconds (90\%) training data. At each level of 
training data size, 20 classification iterations were run for each subject and condition using a new, random training data selection. The mean classification sensitivities for both annotated and non-annotated activities were recorded for each level of training data size. The results of these classifications are demonstrated in Figure 18.

The results in Figure 18 indicate that increases in training data size generally correspond to an improvement in classification performance. For annotated activities, training data sizes less than 60 seconds corresponded to low mean classification sensitivities. As training data size increased to $\sim 90$ seconds, all mean classification sensitivities regardless of condition were greater than $90 \%$.

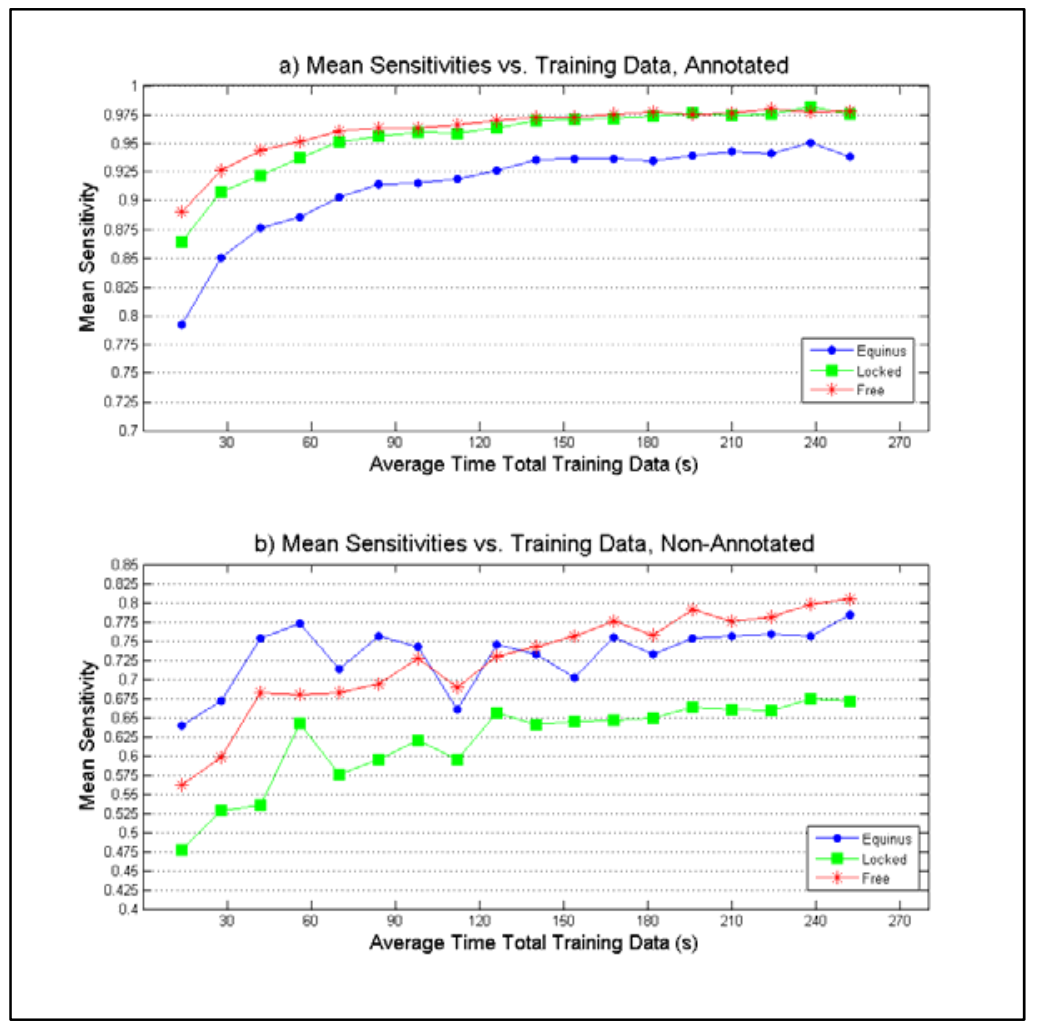

Figure 18. Mean sensitivities as a function of the amount of data used for training for both annotated (a) and non-annotated (b) activity. 
At $\sim 140$ seconds of training data, the framework performance began to plateau, with less than a $2 \%$ increase in mean sensitivity at the maximal inclusion of training data, $\sim 250$ seconds. Limited training data appeared to have a greater effect in the perturbed conditions, as the gaps in sensitivity for Equinus and Locked conditions are larger at minimal training data than at maximal training data. Equinus shows nearly $10 \%$ decrease in sensitivity from Free at 5\% training data, but the discrepancy decreases to less than $4 \%$ at near 59\% training data. Sensitivity for Locked shows less decrease from Free sensitivity at minimal training data, but nearly matches Free at 50\% training data. For non-annotated walking and standing, lower levels of training data led to sporadic results, though apparent leveling occurred around 150 to 190 seconds of training data. This result indicates that training data selection may be more significant when attempting to classify natural movement using lab acquired training data in the form of predefined, controlled movement.

\subsubsection{Sensitivity as a Function of $\mathbf{k}$}

The value of $k=1$ for this study was chosen primarily due to the limited training data for stair use and sitting available. Under the collection protocol, certain activities or postures were underrepresented in the data. Increasing the value of $\mathrm{k}$ would increase the likelihood of including neighbors from incorrect classes, especially if the value of $\mathrm{k}$ exceeded the number of available neighbors from the correct class. The value of $\mathrm{k}$ should be examined more closely, however, since previous work has demonstrated that larger values can provide higher levels of accuracy ${ }^{49}$. For this analysis, 20 iterations for each subject in each condition were run for values of $\mathrm{k}=1,3$, and 5. Figure 19 shows how the 
value of $\mathrm{k}$ affects mean sensitivity values for the recognition of each activity or posture in each condition.

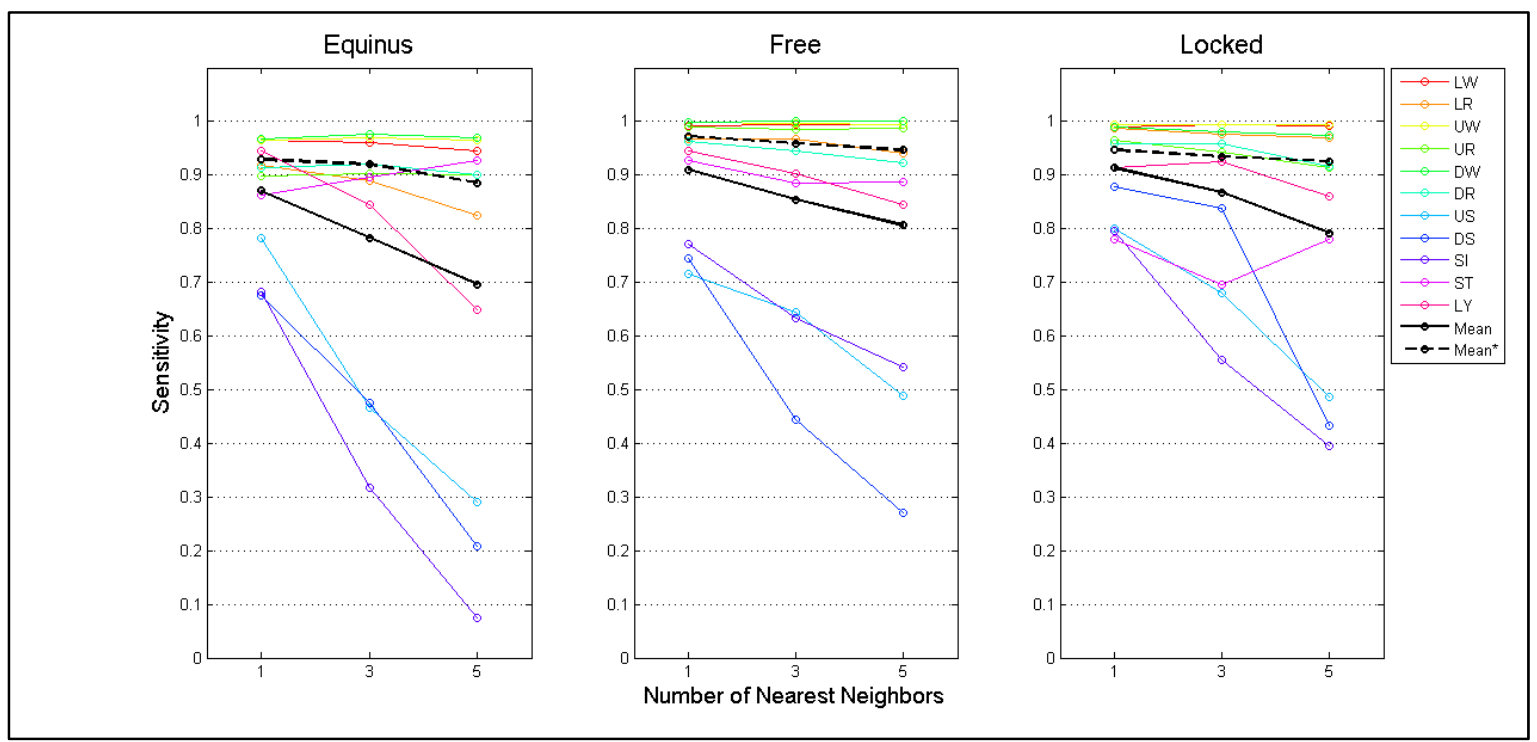

Figure 19. Mean sensitivities of each activity and posture for $\mathrm{k}$ values of 1,3 , and 5 . $\mathbf{L W}$ - level walk; LR - level run; UW - uphill walk; UR - uphill run; DW - downhill walk; DR - downhill run; US - upstairs; DS - downstairs; SI - sitting; ST - standing; LY lying, Mean - Mean of all activities and postures, Mean* - mean without stairs and sitting.

As expected, Figure 19 shows that stair use and sitting recognition suffer as the value of $\mathrm{k}$ increases. Certain activities such as standing show increased recognition in some cases, and several gait activities show peak classifications for a value of $\mathrm{k}=3$. Despite this, mean classification sensitivity decreases with an increasing value of $k$, even when stair use and sitting are not considered.

As data becomes larger in future studies, the value of $\mathrm{k}$ should be heuristically optimized to provide the best results. Generally a higher value of $\mathrm{k}$ should provide better results so long as training data size for each activity is larger than k. Additionally, returning the indices of additional nearest neighbors does not significantly increase 
computational costs, since the distance to each neighbor will have already been calculated in order to determine the nearest neighbor.

\subsubsection{Forced Classification at $k=1,50 \%$ Training Data}

Considering the results discussed in the previous two sections, the classification routine was performed over 20 iterations for all subjects and conditions at $\mathrm{k}=1$ and a random partition of half the annotated data for training ( $\sim 140$ seconds per trial) for each iteration. The value of $k=1$ provided the best sensitivity results, and using half of the annotated data for training allowed near maximal sensitivity results while maintaining a training data set which did not outsize the test data set. The results indicate that this framework is highly successful at recognizing annotated activities and postures. The framework also possesses some ability to classify non-annotated walking and standing using annotated training data. Table 3 provides the mean and standard deviation classification sensitivities for each activity or posture, both annotated and non-annotated, under each condition, while Figure 20 visually presents classification sensitivities and standard deviations for both annotated and non-annotated events.

The classification scheme showed the ability to classify atypical gait with a relatively small amount of training data. Many other studies using machine learning algorithms use subsets of training data which significantly outsized the test data ${ }^{14,18,55}$. The classification sensitivities from this study showed that training data sizes which exceeded test data sizes (i.e. $>50 \%$ training data) are not required in order to make accurate classifications, especially for gait activities. 
Table 3. Mean and standard deviation classification sensitivities for annotated and non annotated activities and postures.

\begin{tabular}{|c|c|c|c|c|c|c|c|c|c|c|c|c|}
\hline & \multicolumn{4}{|c|}{ Sensitivity (\%) - Annotated } & \multicolumn{4}{c|}{ Sensitivity (\%) - Non-Annotated } \\
\cline { 2 - 17 } & \multicolumn{2}{|c|}{ Equinus } & \multicolumn{2}{c|}{ Free } & \multicolumn{2}{c|}{ Locked } & \multicolumn{2}{c|}{ Equinus } & \multicolumn{2}{c|}{ Free } & \multicolumn{2}{c|}{ Locked } \\
\hline & M & SD & M & SD & M & SD & M & SD & M & SD & M & SD \\
\hline Level Walk & 96.5 & 1.6 & 99.1 & 0.5 & 99.1 & 0.8 & 49.0 & 5.4 & 73.1 & 3.9 & 71.4 & 4.8 \\
\hline Level Run & 91.9 & 5.0 & 96.8 & 4.0 & 98.8 & 2.3 & & & & & & \\
\hline Uphill Walk & 96.6 & 1.8 & 99.9 & 0.4 & 99.4 & 0.7 & & & & & & \\
\hline Uphill Run & 89.8 & 5.3 & 99.1 & 1.3 & 96.6 & 3.4 & & & & & & \\
\hline Downhill Walk & 96.8 & 1.4 & 99.9 & 0.4 & 98.9 & 0.9 & & & & & & \\
\hline Downhill Run & 91.5 & 5.2 & 96.3 & 3.8 & 95.8 & 2.9 & & & & & & \\
\hline Upstairs & 78.3 & 15.4 & 71.7 & 9.9 & 80.0 & 9.5 & & & & & & \\
\hline Downstairs & 67.5 & 21.3 & 74.5 & 15.4 & 87.8 & 11.3 & & & & & & \\
\hline Sitting & 68.3 & 17.0 & 77.1 & 11.4 & 79.5 & 14.3 & & & & & & \\
\hline Standing & 86.2 & 5.6 & 92.8 & 5.1 & 78.0 & 8.7 & 75.0 & 13.7 & 77.7 & 7.1 & 65.0 & 5.3 \\
\hline Lying & 94.5 & 6.0 & 94.5 & 3.6 & 91.4 & 6.3 & & & & & & \\
\hline
\end{tabular}

The results also indicate that the framework possesses a strong ability to classify gait activities at various inclines. Mean sensitivities and standard deviations for all activities at $\sim 140 \mathrm{~s}$ training data are shown in Figure 20. All annotated gait events, not including stair use, classified at $\geq 90 \%$ sensitivity, with walking events having the highest sensitivities. The mean classification sensitivity for all walking events regardless of condition and incline was $98.5 \pm 1.9 \%$. Classification of running events was also highly successful, with an overall mean classification sensitivity of $95.1 \pm 3.7 \%$.

At mean sensitivities of $77 \%$ and $78 \%$ respectively, stair climbing and descending events were most poorly recognized, a result consistent with other studies ${ }^{57,61}$. Low sensitivities for these events could be explained in part by limited training data availability. The laboratory staircase only had 4 steps, and subjects generally spent about $10 \mathrm{~s}$ per trial on the stairs. Using $50 \%$ training data provided only 5 windows for training in this case. The results on training data size show in Figure 18 confirm that limited training data compromises sensitivity. Table 3 shows large standard deviations for stair use recognition, at between $9.9 \%$ and $21.3 \%$., indicating that training data selection has a 
significant effect on sensitivity. Coley et al., which was successful in differentiating stair climbing from level walking and stair descending, did not specify how many stair steps were used $^{56}$. Foerster et al. showed difficulty in classifying stair use with 60 steps $^{61}$. Future research should examine if increasing the time of stair use improves sensitivity in classification.

Mean annotated posture recognition sensitivities ranged from $65 \%-95 \%$, with sitting being generally the most poorly classified. Postures also showed large standard deviations. Annotated sitting and standing were classified at mean sensitivities of $75 \%$ and $87 \%$, respectively. This was largely due to confusion between the two. Differentiations in posture are made by comparing shank and foot orientations, and these are often similar for non-reclined postures. Table 4 shows a conglomerate confusion matrix for forced classification of annotated and non-annotated activity and demonstrates how sitting and standing were often confused. Thigh, waist, or sternum markers have improved posture recognition, but they are outside the scope of this study ${ }^{13,14,61}$.

Table 4. Conglomerate confusion matrix for all annotated and non-annotated activities. Includes summation over 20 iterations for all subjects and conditions.

\begin{tabular}{r|ccccccccccc}
\hline & LW & LR & UW & UR & DW & DR & US & DS & SI & ST & LY \\
\hline LW & 9136 & 9 & 44 & 0 & 47 & 0 & 41 & 31 & 3 & 14 & 1 \\
LR & 0 & 1463 & 0 & 18 & 0 & 63 & 0 & 0 & 0 & 0 & 0 \\
UW & 31 & 0 & 6721 & 63 & 0 & 0 & 12 & 5 & 1 & 22 & 0 \\
UR & 0 & 3 & 20 & 2159 & 0 & 0 & 0 & 0 & 0 & 0 & 0 \\
DW & 34 & 12 & 0 & 0 & 5729 & 27 & 0 & 4 & 0 & 0 & 0 \\
DR & 0 & 31 & 0 & 0 & 0 & 1740 & 0 & 0 & 0 & 0 & 0 \\
US & 12 & 0 & 0 & 0 & 11 & 0 & 415 & 52 & 8 & 43 & 3 \\
DS & 13 & 0 & 0 & 0 & 0 & 0 & 23 & 388 & 7 & 14 & 4 \\
SI & 3 & 0 & 0 & 0 & 3 & 0 & 2 & 6 & 426 & 108 & 36 \\
ST & 45 & 0 & 9 & 0 & 0 & 0 & 47 & 14 & 107 & 1460 & 25 \\
LY & 0 & 0 & 0 & 0 & 0 & 0 & 0 & 0 & 8 & 9 & 951 \\
\hline
\end{tabular}


Decreases in sensitivity in annotated activity for perturbed conditions as compared to the Free condition were most prevalent in running and standing events for Equinus and standing events for Locked, as seen in Figure 20. Equinus showed decreases in running sensitivities by an average of $6.2 \%$ from the other two conditions, with an increase in running standard deviation by an average of $2.4 \%$. Figure 20 indicates that Equinus had overall higher standard deviations in sensitivity than Free and Locked. This may be due to excessive "wasteful" movements as those discussed by Herndon et al ${ }^{50}$.

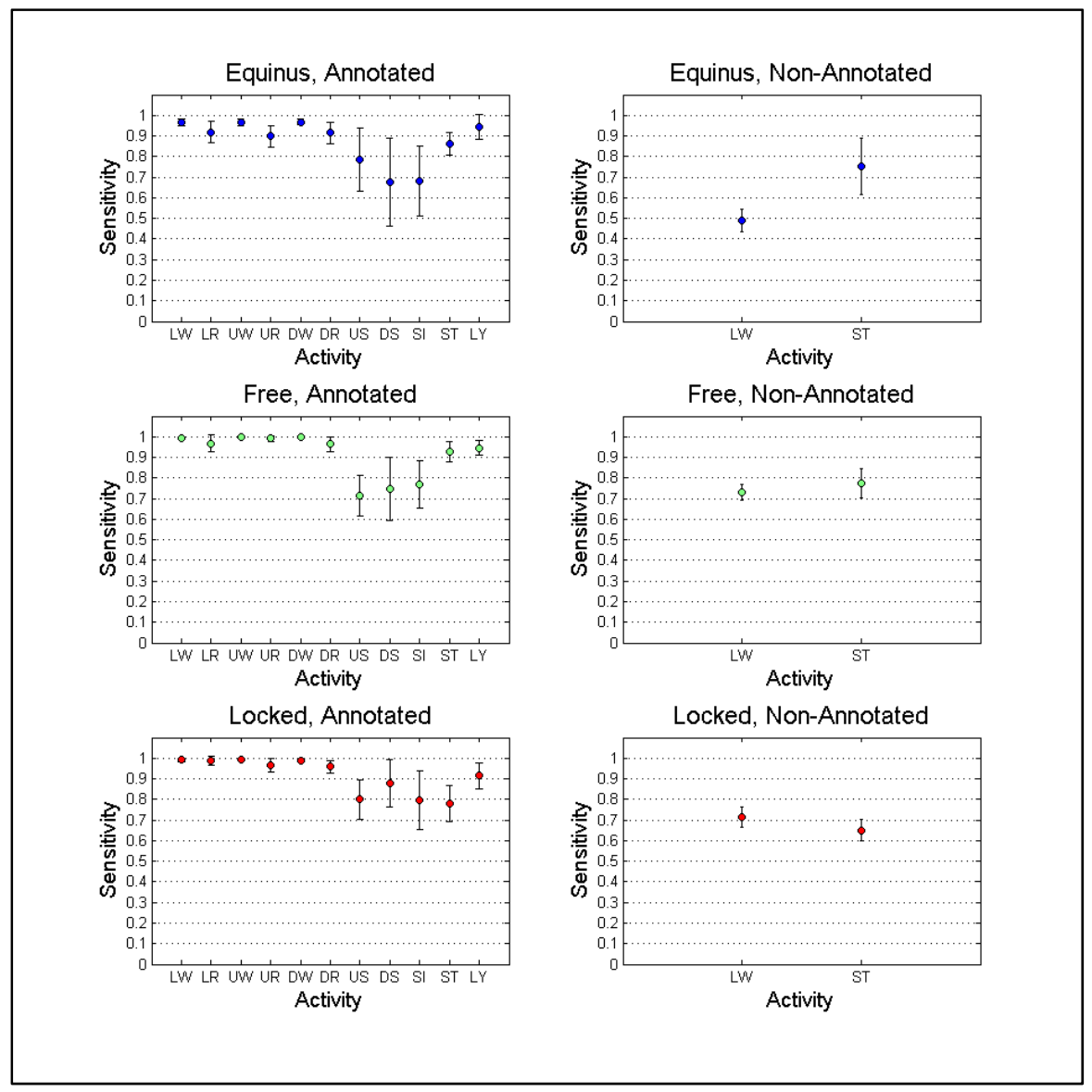

Figure 20. Means and standard deviations of classification sensitivities for annotated and non annotated activities and postures. $\mathbf{L W}$ - level walk; $\mathbf{L R}$ - level run; $\mathbf{U W ~ - ~ u p h i l l ~}$ walk; UR - uphill run; DW - downhill walk; DR - downhill run; US - upstairs; DS downstairs; SI - sitting; ST - standing; LY - lying. 
For standing, Locked showed a decrease in sensitivity by up to $14.8 \%$ from the other conditions, with increases in standard deviation of up 3.6\%. This result was consistent with expectation since the Locked condition by its nature confined the shank and foot angles. Decreases in the mean sensitivities for classification of perturbed activities and postures suggests that the prescribed AFO conditions create a wider array of typical movements for a particular activity. The increased standard deviations in classification sensitivity mirror the higher standard deviation in the training feature sets for the perturbed conditions discussed in Section 3.3.3. These results suggest that training selection was more important for perturbed conditions, especially in Equinus gait activities and Locked postures.

The framework also showed some ability to classify semi natural movements. Sensitivity levels for non-annotated data walking and standing were lower than for annotated data, but were high enough to indicate that the algorithm can potentially classify natural movement using predefined activity for training. For non-annotated walking, mean sensitivity was around $70 \%$ for both Free and Locked, but fell to around $50 \%$ for Equinus. Mean sensitivity for non-annotated standing was around $70 \%$ for all conditions.

Standard deviations in sensitivity for non-annotated events were between 3 and 7 times larger than those for annotated events. The large standard deviations seen in Figure 20 suggest that the selection of training data largely effects how well non-annotated events can be recognized. The large variability in classification of natural movement has been previously discussed in a study by Tapia et al., in which classification accuracies of many natural movements ranged from $7 \%$ to $89 \%{ }^{62}$. 
The subjectivity in ground truth definitions must be discussed when considering semi-natural or natural movement. Recognition of human activity is limited by the continuous nature of human movement. Unlike more discrete areas such as human speech recognition, human movement classification relies on subjective distinction between events in order to enable categorization. For instance, during non-annotated periods, subjects frequently shuffled or milled about in ways that resembled walking or standing, but the distinction between the two was often unclear. How much must a person move to be considered walking? Must a person be completely still to be considered standing? Perhaps "milling around" could be classified as an activity in its own right. Subjectivity of the observer presents a challenge when assessing sensitivity of classifying nonannotated events, and future research must look to refine the boundaries of activity and posture. For clinical cases, the recommendations of the supervision physician or physical therapists may help define classification boundaries.

\subsection{Feature Selection Using Cluster Analysis}

The 23 features described in Section 3.3.2 were all included to provide as complete a physical description of the lower limb kinematics of the AFO user. Some features, however, are irrelevant in certain classification levels. For instance, the FFT coefficients are ineffective when attempting to recognize various postures, and the orientation coefficients may not be suitable for differentiating activity periods, since they show positional changes only at frequencies less than $0.5 \mathrm{~Hz}$. As such, this work considered how the removing certain features at each level of the framework could 
promote accurate classification. This section will describe a feature selection routine which considers the clustering precision of each feature within the training data.

\subsubsection{Clustering in the Training Data}

In a 2005 paper, Huynh and Schiele analyzed the cluster distribution for onedimensional accelerometer derived features, defining a cluster precision for each feature $^{63}$. This thesis work used a similar method to Huynh and Schiele in order to determine which features were most significant at each of the six levels of classification.

For each level of classification, the 23 features were analyzed for cluster precision in the training data. Based on its particular feature magnitude, each training data window was placed into one of a number of clusters, defined by the number of potential classifications in the framework level. For instance, training data for the Active/Static classification level were partitioned into two clusters for each feature, one for activities and one for postures.

The clusters were defined using the standardized Euclidean distance as described in Section 3.4.2. The cluster centers were selected so as to minimize the within-cluster sum of squares distances of the data windows to the cluster centers, that is minimizing

$$
\sum_{j=1}^{l} \sum_{i=1}^{w_{c}}\left\|\frac{x_{i}-c_{j}}{\sigma_{j}^{2}}\right\|^{2}
$$

where $x_{i}$ is the feature magnitude of the $i^{\text {th }}$ data window and $c_{j}$ is the feature magnitude of the proposed cluster center, $\sigma_{j}$ is the standard deviation of the $1-b y-w_{c}$ feature vector for each proposed cluster, $l$ is the number of clusters, and $w_{c}$ is the number of data windows 
in each cluster. Ideally, each cluster would exclusively represent one of the available classifications.

The weighted cluster precision, $p$, of each feature is defined as

$$
p=\sum_{j=1}^{l} \frac{N_{q_{j}}}{N_{b_{j}}} u_{j}
$$

where $N_{q_{j}}$ is the number of data windows accurately grouped within the $j^{\text {th }}$ cluster; $N_{b_{j}}$ is the total number of training data windows which belong to a particular classification, defined by ground truth; $v_{j}$ is the weight prescribed to each cluster, defined as

$$
u_{j}=\frac{\sum M_{j}-M_{j}}{\sum M_{j}}
$$

where $N_{b_{j}}$ is defined as above, giving $\Sigma N_{b_{j}}$ as the total number of training data windows analyzed. The weighting was performed so that the clustering precision favored those activities that had a representatively small number of training data windows, such as stair use or sitting, since these activities were more difficult to classify. The precision values were normalized from 0 to 1 , where a feature with a $p=1$ was able to classify every training data window into its appropriate cluster, and a feature with $p=0$ was unable to correctly classify a single data window.

\subsubsection{Results with Feature Selection}

The researchers evaluated the sensitivity of the framework using the feature selection methods described above. A threshold value, $t$, was set such that any feature 
with a $p \geq t$ would be used for classification at the particular level. The results were compiled by running 20 iterations of the framework with randomized training data selection at threshold levels ranging from 0 to 1 in increments of 0.1 . The sensitivity and the average number of times each feature was used at a particular threshold were recorded.

As discussed in Section 3.3.2, certain features are more or less important depending on which intermediate classification is being performed. Figure 21 shows the usage percentage of each feature over 20 iterations of random training data selection at each selection threshold.

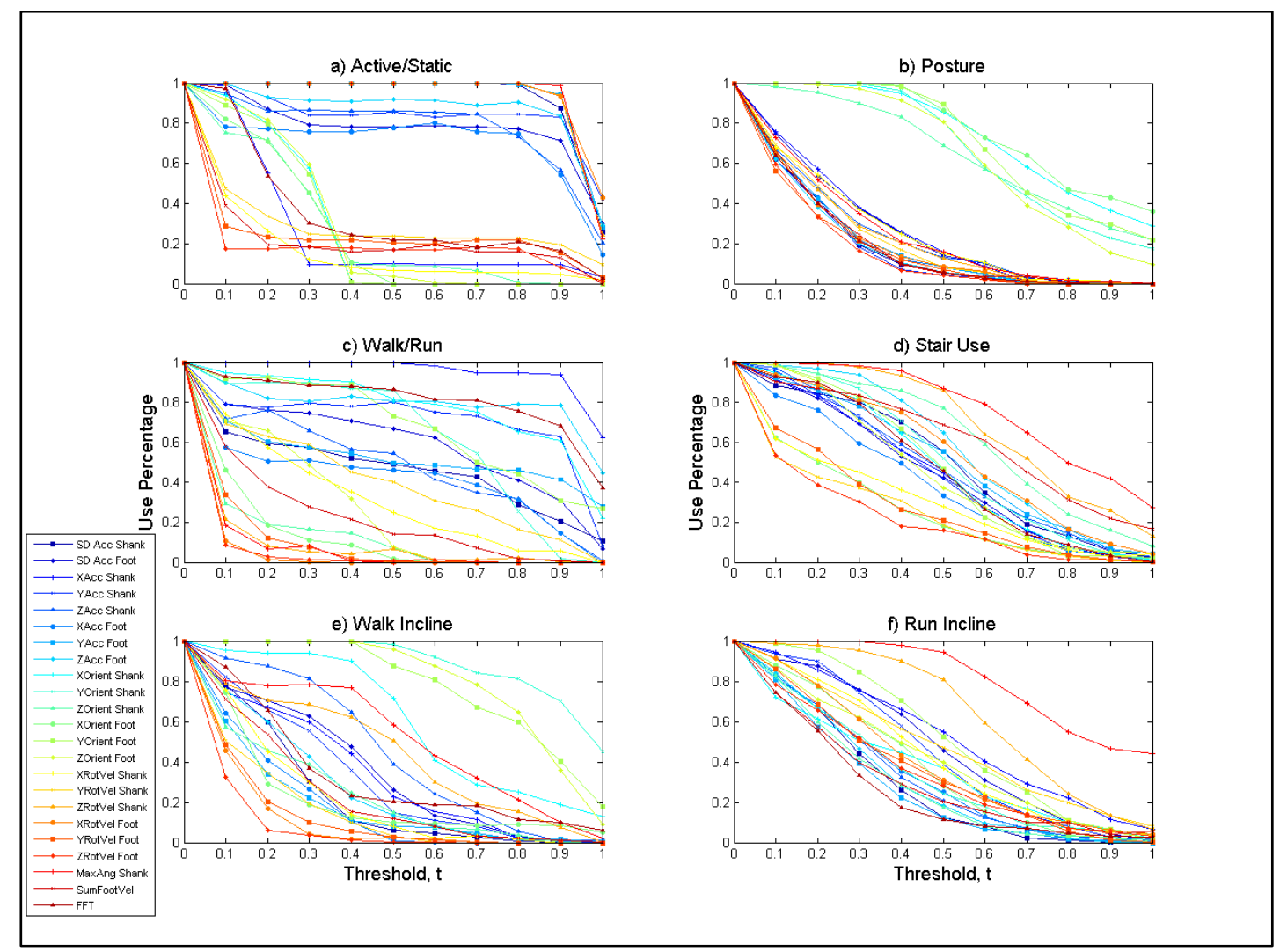

Figure 21. Usage percentage for each of the 23 features at each level of classification as a function of the selection threshold 
It is apparent that for certain levels of classification, certain features carry more significance than others. Figure 21 a shows that the differentiation between activity and static posture can largely be determined by a subset of seven different activities, almost all of which are measures of sensor acceleration, while Figure $21 \mathrm{~b}$ shows that posture recognition depends largely on the orientation components of the accelerometers. For each subsequent level of classification, certain features are used with higher frequently as the precision threshold increases. The importance of feature selection depends heavily on if classifications are being performed on supervised or unsupervised movement data. As can be seen in Figure 22, sensitivity for classification of annotated activity steadily declined as features were removed for all conditions. For non-annotated walking and standing, however, the results indicate that feature selection may be useful.

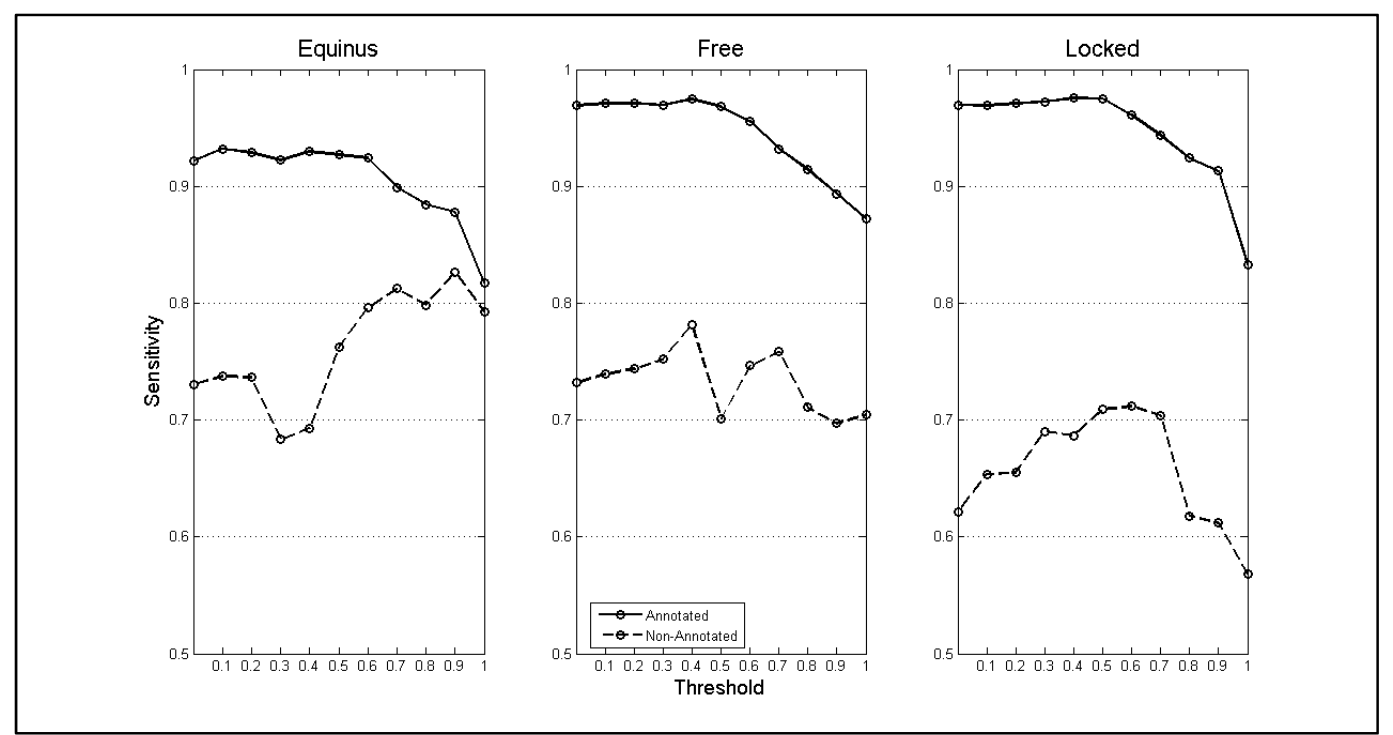

Figure 22. Mean sensitivities for annotated activities and non-annotated walking and standing walking as compared to the precision threshold for feature selection.

Maximum sensitivities were obtained at different points for each condition, with the Equinus condition preferring the most selective feature threshold of the three 
conditions, with sensitivity maximized at $t=0$.9. The Locked condition showed maximal sensitivity at $t=0.6$. The Free condition had maximal mean sensitivity at $t=0.4$, and showed the smallest increase in sensitivity of the three from $t=0$ to the point of maximal sensitivity.

The results indicate that feature selection can provide better results when attempting to classify natural movement using predefined movement for training, especially for conditions like Equinus which show inconsistencies in gait parameters. Maurer et al. showed similar results, indicating that features which are highly correlated within classification groups can be removed without reducing accuracy ${ }^{57}$. Huynh and Schiele similarly showed that for classification of specific activities, removal of nondescriptive features could improve recognition ${ }^{63}$.

\subsection{Introducing a Declassification Distance Metric}

While the results discussed in Sections 3.5 and 3.6 provide a comprehensive view of the sensitivity of the classification framework, they are based entirely on the occurrence of true positives in classification. They do not consider the rate of false positives. In clinical applications of this framework, false positives can misinform diagnosticians as to the overall activity level or particular ability of the AFO user. This can have very serious implications when treatment options are considered. To reduce the incident of false positives during classification, a distance metric was designed which would enable the framework to declassify events which did not closely resemble any of the predefined movements. 


\subsubsection{Calculation of the Distance Metric}

The distance metric was designed to reduce the incidence of false positives without upsetting true positive recognition. In order to assess classification results using the distance metric, specificity is introduced in the results, defined as

$$
\text { Specificity }=\frac{T N}{T N+F P}
$$

where $T N$ is the number of true negatives and $F P$ is the number of false positives. The addition of the distance metric to the framework allowed for the declassification of any test data window whose distance from its nearest neighbor was a statistical outlier from the training data. At each of the six levels in the framework, the set of training data for that classification, represented by $\boldsymbol{X}_{\boldsymbol{b}}$, the $w_{b}-b y-m$ matrix where $w_{b}$ is the particular number of windows in that set, and $m$ is the number of features (23). Each level contained a number of classification subsets, $N_{s}$ (e.g. in the Active/Static level, all activity periods were split into one subset and all static periods into another, $N_{s}=2$ ). For each subset, the coordinate standardized Euclidean distances were calculated between each training data window and all remaining training data windows in the same manner as in equations (3) - (5). For each classification level, the number of distances, $N_{d}$, calculated was:

$$
N_{d}=\frac{m}{2} \sum_{s=1}^{N_{s}} w_{b}\left(w_{b}-1\right)
$$


Outliers for each subset were defined by using the interquartile range of the distances. Any test data window whose distance was found to be a statistical outlier in this sense was declassified:

$$
D_{\text {outlier }}>Q_{3}+h\left(Q_{3}-Q_{1}\right)
$$

where $Q_{1}$ and $Q_{3}$ are the $25^{\text {th }}$ and $75^{\text {th }}$ percentile, respectively, of the training data distances and $h$ is a scaling constant. A low scaling constant generates more declassifications. Different values of $h$ were considered, and the optimal scaling constant was determined heuristically at each level in a manner which maximized the sensitivity and specificity of the classifications.

\subsubsection{Results with Varied Scaling Constant}

The value of the scaling constant has a direct effect on the classification accuracy. A scaling constant which is too small will generate a large number of false negatives, reducing sensitivity, while a scaling constant which is too large will generate a large number of false positives, reducing specificity. The scaling constants must be chosen to optimize the results in a clinically beneficial manner. If false negatives are deemed less consequential than false positives, a lower scaling constant should be selected to maximize specificity. Otherwise a larger scaling constant should be selected to maximize sensitivity.

Figure 23 shows the changes in mean sensitivity and specificity as the scaling factor was adjusted for non-annotated walking and standing. Results in terms of specificity were particularly useful when considering the non-annotated data 
representative of semi-natural movement, as annotated data was generally not prone to a high rate of false positives. It is seen from Figure 23 that, as expected, specificity decreases as the declassification threshold increases, as this will allow a greater number of false positives. Conversely, sensitivity increases with the threshold, since a low threshold promotes the occurrence of false negatives.

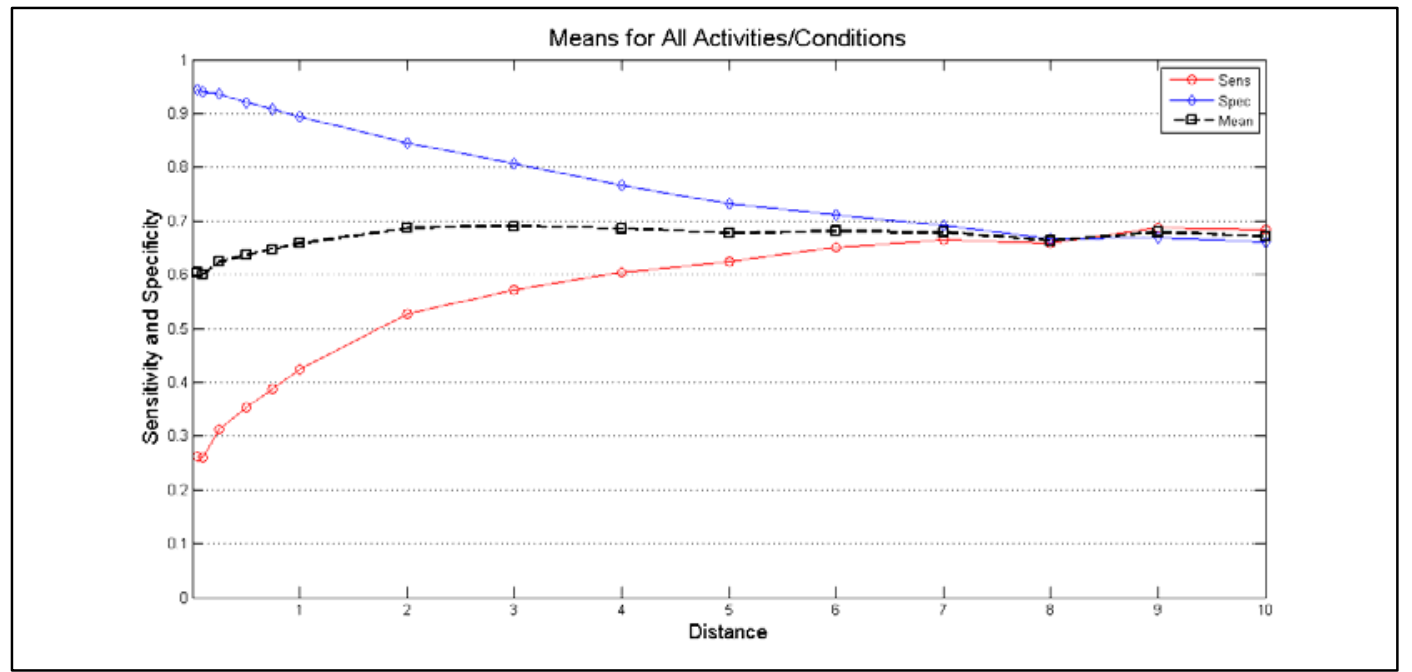

Figure 23. Mean sensitivity and specificity values for non-annotated standing and walking with varying declassification threshold scaling factor. 'Mean' represents the mean of the sensitivity and specificity.

Examining the mean of sensitivity and specificity, a peak is observed when the scaling factor is at a value of 3 , though the mean remains relatively constant with an increasing scaling factor. This suggests that any declassification threshold greater than $Q_{3}$ plus three times the IQR can be used, depending on the sensitivity or specificity requirements of the case.

\subsubsection{Intermediate Classification}

The distance metric also allows intermediate classifications. In some cases, especially when considering semi-natural movement, the coordinate distance of the test 
window may remain under the declassification threshold for only 1 or 2 classification levels. For instance, the framework may recognize that the subject is static, but cannot discern the posture. Or perhaps the subject is walking, but the grade is unknown.

Intermediate classifications can provide at least some description of activity even if a full picture is unavailable. In addition, intermediate classifications can lead to the formation of new semi-classified clusters within the feature space. These clusters can be considered as new activity or posture forms in which the subject frequently engages. Figure 24 displays what intermediate classifications may look like, and where new clusters can potentially be found.

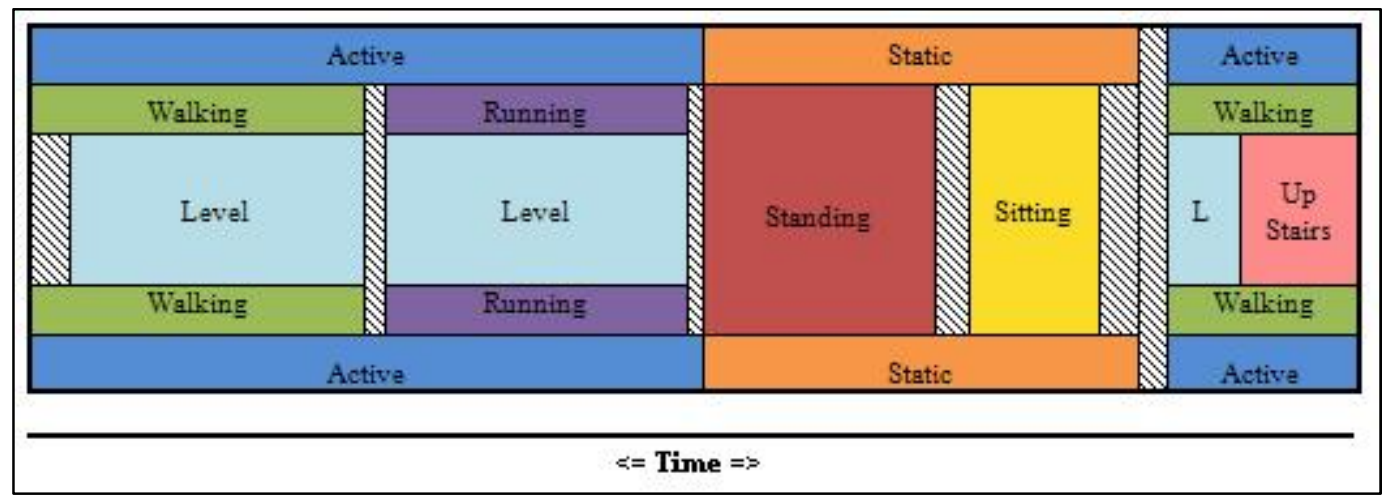

Figure 24. Example of intermediate classifications. The most basic classification of Active/Static is followed by intermediate classifications of posture recognition and/or gait activity, which are further classified into inclines or stair use. The hashed areas represent

places where classifications could not be made at a particular level, and only an intermediate classification or no classification is made.

In places where sub-level classifications cannot be made, marked by the hashed areas in Figure 24, new data clusters may arise which represent activity or posture previously undefined within the framework. The recognition of new clusters can allow researchers to expand the framework include new sub-classifications. This will be 
particularly important when assessing natural movement in different subjects, where pathology or personal preference characterize a variety of activity and posture forms. 


\subsection{Conclusions and Recommendations for Future Work}

The prevalence of AFO use in CP patients has opened the door for an advance in mobile, unsupervised gait analysis using lower limb kinematics. The University of Virginia's Center for Wireless Health has created in TEMPO a device which can be discreetly integrated into an AFO to provide a comprehensive kinematic description of the foot and shank movement. This research introduced a framework for accurately classifying gait activities and postures using AFO-mounted TEMPO sensor data.

\subsection{Summary of Research}

The present research began by carrying out an activity test protocol for eight healthy subjects with perturbed gait through to the use of the custom AFOs. With the data from this test protocol, a set of kinematic features were derived based on recommendation from previous studies and a heuristic analysis of the kinematic test data. Following the compilation of the 23 kinematic features, a classification routine was developed to maximize sensitivity during forced classification of the eleven prescribed gait activities and postures. The framework, consisting of six levels of 1-nearest neighbor classification, was first used to assess sensitivity results in classification using varying amounts of training data. The results of this test showed that optimal sensitivities could be obtained using training data sets which did not outsize the test data, and that using as little as $140 \mathrm{~s}$ of training data could provide mean classification sensitivities of greater than $90 \%$ for more than 4.5 minutes of annotated data, and up to $75 \%$ for non-annotated 
data of more than 15 minutes. After assessing training data size effects, the number of nearest neighbors was varied at $\mathrm{k}=1,3,5$. The results indicated that a $1-\mathrm{NN}$ classification consistently provided the best sensitivity for classification. The results of this analysis are difficult to interpret, however, since small training data sizes for a number of activities limited the efficacy of larger k classifications. Following the analysis of training data size and k-value, a forced classification routine was carried out using the 23 feature, $1-\mathrm{NN}$ framework with a 50\% random training data partition ( $\sim 140 \mathrm{~s}$ per trial). The forced classification showed very high sensitivity results for annotated gait activities, with decreases in sensitivity for posture recognition and stair use detection. The forced classification routine also showed only a small decrease in mean sensitivity of classification for the atypical conditions, Equinus and Locked, but showed a significant increase in the standard deviation of the classifications for these conditions. The results also indicated that assessing non-annotated data using annotated training data is feasible, though decreases in sensitivity were found. The work specifically highlighted how atypical gait conditions can limit classification of semi-natural movement using predefined activity for training. Feature selection using clustering means was then tested, indicating that classification of semi-natural or natural movement using predefined training data can benefit from selecting features which are most relevant to intermediate classifications, especially for inconsistent gait patterns. The work then introduced a declassification metric which weighed the results in terms of sensitivity and specificity, showing that the declassification of activity outliers can increase specificity of the framework. 


\subsection{Recommendations for Future Work}

Body sensor networks using IMUs have incredible diagnostic potential. This work studied the capability of lower limb mounted IMUs in the application of activity recognition of AFO users. The results of this thesis provide a valuable stepping stone for future work in implementing AFO mounted sensor networks.

The system has shown the ability to use subject-specific training data to classify atypical gait patterns, but other studies have developed algorithms for healthy subjects which do not require subject-specific training, often making use of Bayesian adaptations $^{13,14}$. With the large amount of variability present in pathological gait, however, inter-subject training may not be feasible. Therefore, this study focused on activity classification using subject-specific training. Future work should study gait pattern variability in AFO users to potentially develop a cross-subject training algorithm.

It has yet to be shown how the classification algorithm would perform in a completely natural setting, where the number and permutation of gait activities and postures notably increase. Particular challenges may appear in marking various postures using only shank and foot mounted sensors and in noting the presence of incline at variable grades. Future studies must focus on classification of activity in actual AFO users in natural environments.

Data collection protocols should be altered to include more training data windows for each activity. Specifically, future studies should ensure protocols allow for sufficient collection of annotated activity periods so that the value of $\mathrm{k}$ can be adjusted to maximize sensitivity and specificity results without being limited by small training data size. 
Remarkably, kNN algorithms do not scale well to large training data sizes since the distances between each test datum and all training data must be calculated. They become more computationally expensive as the amounts of training data and feature size grow. With the recommendation described in the above paragraph, training data size should be monitored with computational costs in mind. While this work showed that classifications with high sensitivity cold be made even with small sets of training data, future work should explore regression-based algorithms, such as random forest, which are less affected by data size, as it is yet unclear how much training data is necessary to classify daily natural activity.

This research was an integral step in the enabling of mobile gait monitoring in $\mathrm{CP}$ patients. It provides a basis for activity classification in AFO users, and the classification framework can be used in several applications. In the immediate future, the framework should be used and optimized for classifying natural movement in healthy individuals wearing custom AFOs using annotated predefined activity for training. The framework can be used to assess the efficacy of training data collection protocols in natural activity situations. The next step will be to optimize the framework for actual CP patients who wear AFOs. Special consideration should be given to training methods and recognition of previously undefined gait and posture forms. This research is most significant in that it enables the determination of the spatio-temporal parameters of gait which inform the diagnostic choices of the patient's care givers. Periods of walking must be identified within a patient's regular daily movement to enable the acquisition of relevant parameters such as stride length, cadence, and foot contact time. Future work should seek to use the activity classifications and AFO mounted sensor data to determine these relevant gait 
parameters. A robust mobile system which could identify periods of walking and accurately define gait parameters would enable caregivers and patients to confidently assess the costs and benefits of available treatment options, and at a fraction of the cost of optical motion capture systems. 


\section{Bibliography}

1. Bax, M., Goldstein, M., Rosenbaum, P., Leviton, A. \& Paneth, N. Proposed definition and classification of cerebral palsy, April 2005. Developmental Medicine \& Child Neurology 47, 571-571 (2005).

2. Cerebral Palsy Fact Sheet. (2013). at < http://www.ucpcm.org/uploads/media_items/cp-fact-sheet.original.pdf_>

3. Papavasiliou, A. S. Management of motor problems in cerebral palsy: A critical update for the clinician. European Journal of Paediatric Neurology 13, 387-396 (2009).

4. Carlson, W. E., Vaughan, C. L., Damiano, D. L. \& Abel, M. F. Orthotic management of gait in spastic diplegia. Am J Phys Med Rehabil 76, 219-225 (1997).

5. Lucareli, P. R. G., Lima, M. D., Lucarelli, J. G. D. \& Lima, F. P. S. Changes in joint kinematics in children with cerebral palsy while walking with and without a floor reaction ankle-foot orthosis. Clinics 62, 63-68 (2007).

6. Westberry, D. E. et al. Impact of ankle-foot orthoses on static foot alignment in children with cerebral palsy. The Journal of Bone and Joint Surgery. American volume 13 (2007).

7. Bennett, B. C. et al. Center Of Mass Analysis Of Gait In Children With Cerebral Palsy With And Without Ankle Foot Orthoses. Gait \& Clinical Movement Analysis Society (2005).

8. Abel, M. F. et al. Relationships among musculoskeletal impairments and functional health status in ambulatory cerebral palsy. Journal of Pediatric Orthopaedics 23, 535-541 (2003).

9. Knutson, L. M. \& Clark, D. E. Orthotic Devices for Ambulation in Children with Cerebral-Palsy and Myelomeningocele. Physical Therapy 71, 947-960 (1991).

10. Perry, J. Gait Analysis: Normal and Pathological Function. (SLACK, Inc., 1992).

11. Wren, T. A. L., Rethlefsen, S. \& Kay, R. M. Prevalence of specific gait abnormalities in children with cerebral palsy - Influence of cerebral palsy subtype, age, and previous surgery. Journal of Pediatric Orthopaedics 25, 79-83 (2005).

12. Morris, C. Orthotic management of cerebral palsy. Developmental Medicine and Child Neurology 49, 791-796 (2007).

13. Allen, F. R., Ambikairajah, E., Lovell, N. H. \& Celler, B. G. Classification of a known sequence of motions and postures from accelerometry data using adapted Gaussian mixture models. Physiol. Meas. 27, 935-951 (2006).

14. Bao, L. \& Intille, S. S. in Pervasive Computing, Proceedings (Ferscha, A. \& Mattern, F.) 3001, 1-17 (2004).

15. Ermes, M., Parkka, J., Mantyjarvi, J. \& Korhonen, I. Detection of daily activities and sports with wearable sensors in controlled and uncontrolled conditions. Ieee Transactions on Information Technology in Biomedicine 12, 20-26 (2008).

16. Mathie, M. J., Celler, B. G., Lovell, N. H. \& Coster, A. C. F. Classification of basic daily movements using a triaxial accelerometer. Medical \& Biological Engineering \& Computing 42, 679-687 (2004). 
17. Najafi, B. et al. Ambulatory system for human motion analysis using a kinematic sensor: Monitoring of daily physical activity in the elderly. IEEE Transactions on Biomedical Engineering 50, 711-723 (2003).

18. Parkka, J. et al. Activity classification using realistic data from wearable sensors. Ieee Transactions on Information Technology in Biomedicine 10, 119-128 (2006).

19. Veltink, P. H., Bussmann, H. B., De Vries, W., Martens, W. L. \& Van Lummel, R. C. Detection of static and dynamic activities using uniaxial accelerometers. IEEE Transactions on Rehabilitation Engineering: A Publication of the IEEE Engineering in Medicine and Biology Society 4, 375-85 (1996).

20. Aminian, K., Najafi, B., Bnla, C., Leyvraz, P.-F. \& Robert, P. Spatio-temporal parameters of gait measured by an ambulatory system using miniature gyroscopes. Journal of Biomechanics 35, 689-699 (2002).

21. Bussmann, H. B. J., Reuvekamp, P. J., Veltink, P. H., Martens, W. L. J. \& Stam, H. J. Validity and reliability of measurements obtained with an 'activity monitor' in people with and without a transtibial amputation. Physical Therapy 78, 989-998 (1998).

22. Bussmann, J. B. J., Van de Laar, Y. M., Neeleman, M. P. \& Stam, H. J. Ambulatory accelerometry to quantify motor behaviour in patients after failed back surgery: a validation study. Pain 74, 153-161 (1998).

23. Paraschiv-Ionescu, A., Buchser, E. E., Rutschmann, B., Najafi, B. \& Aminian, K. Ambulatory system for the quantitative and qualitative analysis of gait and posture in chronic pain patients treated with spinal cord stimulation. Gait \& Posture 20, 113-125 (2004).

24. Moore, S. T., MacDougall, H. G. \& Ondo, W. G. Ambulatory monitoring of freezing of gait in Parkinson's disease. Journal of Neuroscience Methods 167, 340-348 (2008).

25. Salarian, A., Russmann, H., Vingerhoets, F. J. G., Burkhard, P. R. \& Aminian, K. Ambulatory monitoring of physical activities in patients with Parkinson's disease. IEEE Transactions on Biomedical Engineering 54, 2296-2299 (2007).

26. Ng, A. V. \& Kent-Braun, J. A. Quantitation of lower physical activity in persons with multiple sclerosis. Medicine and Science in Sports and Exercise 29, 517 (1997).

27. Mackey, A. H., Stott, N. S. \& Walt, S. E. Reliability and validity of an activity monitor (IDEEA) in the determination of temporal-spatial gait parameters in individuals with cerebral palsy. Gait \& Posture 28, 634-639 (2008).

28. Mackey, A. H., Hewart, P., Walt, S. E. \& Stott, N. S. The sensitivity and specificity of an activity monitor in detecting functional activities in young people with cerebral palsy. Archives of Physical Medicine and Rehabilitation 90, 1396-1401 (2009).

29. Mansfield, A. \& Lyons, G. M. The use of accelerometry to detect heel contact events for use as a sensor in FES assisted walking. Medical Engineering \& Physics 25, 879-885 (2003).

30. Chen, S., Cunningham, C. L., Bennett, B. C. \& Lach, J. Enabling longitudinal assessment of ankle-foot orthosis efficacy for children with cerebral palsy. in Proceedings of the 2nd Conference on Wireless Health 1-10 (ACM, 2011). 
31. Barth, A. T., Hanson, M. A., Powell, H. C. \& Lach, J. TEMPO 3.1: A Body Area Sensor Network Platform for Continuous Movement Assessment. International Conference on Body Sensor Networks: 71-76 (2009).

32. Powell, H. C., Hanson, M. A. \& Lach, J. A Wearable Inertial Sensing Technology for Clinical Assessment of Tremor. Circuits and Systems Conference. BIOCAS: 9-12 (2007).

33. Hanson, M. A., Powell, H. C., Barth, A. T., Lach, J. \& Brandt-Pearce, M. Neural Network Gait Classification for On-Body Inertial Sensors. Sixth International Workshop on Body Sensor Networks 181-186 (3) (2009).

34. Q.Li, J.A.Stankovic, M.Hanson, A.Barth \& J.Lach. Accurate, Fast Fall Detection Using Gyroscopes and Accelerometer-Derived Posture Information. International Workshop on Body Sensor Networks 138-143 (2009).

35. Chen, S., Cunningham, C. L., Lach, J. \& Bennett, B. C. Extracting spatio-temporal information from inertial body sensor networks for gait speed estimation. International Conference on Body Sensor Networks 71-76 (IEEE, 2011).

36. Lester, J., Choudhury, T., Kern, N., Borriello, G. \& Hannaford, B. A hybrid discriminative/generative approach for modeling human activities. in Proceedings of the 19th International Joint Conference on Artificial Intelligence 766-772 (2005).

37. Lester, J., Choudhury, T. \& Borriello, G. A practical approach to recognizing physical activities. Pervasive Computing 1-16 (2006).

38. Huynh, T. \& Schiele, B. Towards less supervision in activity recognition from wearable sensors. 10th IEEE International Symposium on Wearable Computers, 3-10 (IEEE, 2006).

39. Lau, H. Y., Tong, K. Y. \& Zhu, H. Support vector machine for classification of walking conditions using miniature kinematic sensors. Medical and Biological Engineering and Computing 46, 563-573 (2008).

40. Goulermas, J. Y., Howard, D., Nester, C. J., Jones, R. K. \& Ren, L. Regression techniques for the prediction of lower limb kinematics. Journal of Biomechanical Engineering 127, 1020 (2005).

41. Goulermas, J. Y. et al. An instance-based algorithm with auxiliary similarity information for the estimation of gait kinematics from wearable sensors. IEEE Transactions on Neural Networks 19, 1574-1582 (2008).

42. Quinlan, J. R. Induction of decision trees. Machine learning 1, 81-106 (1986).

43. Quinlan, J. R. C4. 5: Programs for Machine Learning. 1, (Morgan Kaufmann, 1993).

44. Breiman, L., Friedman, J., Stone, C. J. \& Olshen, R. A. Classification and Regression Trees. (Chapman \& Hall/CRC, 1984).

45. Reynolds, D. Gaussian mixture models. Encyclopedia of Biometric Recognition 1217 (2008).

46. Rabiner, L. R. A tutorial on hidden Markov models and selected applications in speech recognition. Proceedings of the IEEE 77, 257-286 (1989).

47. Rabiner, L. \& Juang, B. An introduction to hidden Markov models. ASSP Magazine, IEEE 3, 4-16 (1986).

48. Abraham, A. Artificial neural networks. Handbook of Measuring System Design (2005). 
49. Altun, K., Barshan, B. \& Tuncel, O. Comparative study on classifying human activities with miniature inertial and magnetic sensors. Pattern Recognition 43, 3605-3620 (2010).

50. Herndon, S. K., Bennett, B. C., Wolovick, A., Filachek, A., Gaesser, G. A., Weltman, A. \& Abel, M. F. Center of mass motion and the effects of ankle bracing on metabolic cost during submaximal walking trials. Journal of Orthopaedic Research 24, 2170-2175 (2006).

51. Bennett, B. C., Abel, M. F., Wolovick, A., Franklin, T., Allaire, P. E., Kerrigan D. C. Center of Mass Movement and Energy Transfer During Walking in Children With Cerebral Palsy. Archives of Physical Medicine \& Rehabilitation 86, 2189-2194 (2005).

52. Waters, R. L., Lunsford, B. R., Perry, J. \& Byrd, R. Energy-speed relationship of walking: standard tables. Journal of Orthopaedic Research 6, 215-222 (1988).

53. Bell, K. J., Ounpuu, S., DeLuca, P. A. \& Romness, M. J. Natural progression of gait in children with cerebral palsy. Journal of Pediatric Orthopaedics 22, 677-682 (2002).

54. Damiano, D. L. \& Abel, M. F. Relation of gait analyses to gross motor function in cerebral palsy. Developmental Medicine and Child Neurology 38, 389-396 (1996).

55. Maurer, U., Smailagic, A., Siewiorek, D. P. \& Deisher, M. Activity recognition and monitoring using multiple sensors on different body positions. International Workshop on Wearable and Implantable BSNs (IEEE, 2006).

56. Coley, B., Najafi, B., Paraschiv-Ionescu, A. \& Aminian, K. Stair climbing detection during daily physical activity using a miniature gyroscope. Gait \& Posture 22, 287-294 (2005).

57. Maurer, U., Rowe, A., Smailagic, A. \& Siewiorek, D. in Ambient Intelligence in Everday Life (Cai, Y. \& Abascal, J.) 3864, 86-102 (Springer-Verlag Berlin, 2006).

58. Luinge, H. J. \& Veltink, P. H. Measuring orientation of human body segments using miniature gyroscopes and accelerometers. Medical and Biological Engineering and Computing 43, 273-282 (2005).

59. Roetenberg, D., Luinge, H. J., Baten, C. T. M. \& Veltink, P. H. Compensation of magnetic disturbances improves inertial and magnetic sensing of human body segment orientation. IEEE Transactions on Neural Systems and Rehabilitation Engineering 13, 395-405 (2005).

60. Favre, J., Jolles, B. M., Siegrist, O. \& Aminian, K. Quaternion-based fusion of gyroscopes and accelerometers to improve 3D angle measurement. Electronics Letters 42, 612-614 (2006).

61. Foerster, F., Smeja, M. \& Fahrenberg, J. Detection of posture and motion by accelerometry: a validation study in ambulatory monitoring. Computers in Human Behavior 15, 571-583 (1999).

62. Tapia, E., Intille, S. \& Larson, K. Activity recognition in the home using simple and ubiquitous sensors. Pervasive Computing 158-175 (2004).

63. Huynh, T. \& Schiele, B. Analyzing features for activity recognition. Proceedings of the 2005 Joint Conference on Smart Objects and Ambient Intelligence: 159-163 (ACM, 2005). 